\title{
Il nuovo reato di false comunicazioni sociali
}

\author{
Lorenzo Maria Corvucci
}

\begin{abstract}
Riassunto
L'Autore esamina il nuovo reato di false comunicazioni sociali introdotto dalla Legge 27 maggio 2015, n. 69 - in vigore dal 14 giugno 2015 .

In considerazione delle modifiche apportate al nuovo reato di falso in bilancio - che riassume nei suoi elementi costitutivi per evidenziare il novum - focalizza l'attenzione su una questione specifica di particolare importanza, già oggetto di pronunce di legittimità discordanti in seno alla sezione $\mathrm{V}$ della Corte di cassazione, competente per materia, a pochi mesi dalla entrata in vigore della novella.

La questione riguarda se il falso c.d. valutativo sia tuttora punibile, atteso che la nuova disciplina ha circoscritto l'oggetto della condotta attiva ai soli "fatti materiali rilevanti non rispondenti al vero" ovvero all'omissione di "fatti materiali rilevanti la cui comunicazione è imposta dalla legge sulla situazione economica, patrimoniale o finanziaria della società o del gruppo al quale la stessa appartiene", eliminando dunque ogni riferimento alle valutazioni contenute nel testo previgente e provvedendo contestualmente a replicarla anche nella definizione di quello della condotta omissiva, in relazione alla quale le due norme incriminatrici (artt. 2621 e 2622 cod. civ.) in precedenza evocavano le "informazioni" oggetto di omessa comunicazione.
\end{abstract}

\section{Résumé}

L'auteur examine le nouveau crime de fausses informations sur les sociétés institué par la loi nº9 du 27 mai 2015 , entrée en vigueur le 14 juin 2015.

En considération des modifications apportées au nouveau crime de faux en écritures comptables (expliqué de façon synthétique afin de mettre en évidence les nouveautés), l'attention se porte sur une question primordiale, discutée auparavant par la Cinquième Section de la Cour de Cassation, quelques mois après l'entrée en vigueur de la nouvelle loi.

Reste à savoir si le faux d'évaluation est toujours punissable, car la nouvelle loi a limité l'objet de la conduite criminelle à « faits matériels pertinents non conformes à la vérité » ou à l'omission de « faits matériels pertinents dont la communication est imposée par la loi sur la situation économique, financière de la société ou du groupe auquel la société appartient ». Toutes références faites aux évaluations prévues par le texte précédent sont ainsi supprimées.

La nouvelle définition concernant le comportement par omission rappelle les règles précédentes (articles $\mathrm{n}^{\circ} 2621$ et $\mathrm{n}^{\circ} 2622$ du Code Civil italien).

\section{Abstract}

The author examines the new offence of false social communication introduced by the Italian law dated 27 may 2015 n.69 in force from 14 June 2015.

Considering the modifications added to the new offence of false accounting- basically explained to highlight the novum - the attention is paid on a specific major issue, previously discussed by the fifth section of the Italian Supreme Court competent in this matter after a few months from the moment the new law came in force.

The questions applies to the fact whether the fraudulent evidence should remain to be punishable as the new discipline has limited the object of the criminal conduct only to "material relevant facts which are untrue" or to the omission of material relevant facts whose communication is imposed by the law regulating the economic situation, the assets and financial position of the company or of the group to which the company belongs. In this way any reference to the evaluations contained in the text previously in force is eliminated.

Omissive conduct is the new definition recalling the two previous rules (art. 2621 and 2622 of the Italian civil code).

Key words: new offence; false social communication; Italian Law nº9/2015; false accounting; omissive conduct.

\footnotetext{
- E' iscritto all'Ordine degli Avvocati di Bologna dal 1999. Iscritto all'Albo Speciale degli Avvocati Cassazionisti dal 2012. Inserito nell'elenco dei professionisti disponibili alla turnazione per le difese d'ufficio, ai sensi dell'art. 29 d.1. 271/89, in base al requisito della competenza esclusiva in materie penalistiche. Iscritto nello speciale elenco dei difensori d'ufficio in materi di immigrazione ed asilo. Iscritto nell'elenco dei professionisti disponibili alla turnazione per le difese d'ufficio avanti il Tribunale per i minorenni, ai sensi dell' art. 15 c. 1 e 2 d.l. 272/89. Autore di pubblicazioni ed articoli in materie penalistiche, collabora con la Rivista di Criminologia, Vittimologia e Sicurezza, di cui è Componente del Comitato editoriale. Presidente del Collegio dei Revisori dei Conti dell'Università Popolare "Enrico Ferri". Docente in corsi e seminari presso l’Universita' Popolare "Enrico Ferri".
} 


\section{Il testo della novella.}

Con legge 27 maggio 2015, n. 69 (in Gazz. Uff., 30 maggio 2015, n. 124) - Disposizioni in materia di delitti contro la pubblica amministrazione, di associazioni di tipo mafioso e di falso in bilancio - è stata nuovamente riformata la disciplina relativa al delitto di falso in bilancio (artt. 2621 e segg. c.c.).

Questa la nuova disciplina in vigore dal 14 giugno 2015:

"Capo II - Disposizioni penali in materia di società e consorzi.

Articolo n.9 - Modifica dell'articolo 2621 del codice civile.

1. L'articolo 2621 del codice civile è sostituito dal seguente: «Art. 2621 (False comunicazioni sociali). - Fuori dai casi previsti dall'art. 2622, gli amministratori, i direttori generali, i dirigenti preposti alla redazione dei documenti contabili societari, i sindaci e i liquidatori, i quali, al fine di conseguire per sè o per altri un ingiusto profitto, nei bilanci, nelle relazioni o nelle altre comunicazioni sociali dirette ai soci o al pubblico, previste dalla legge, consapevolmente espongono fatti materiali rilevanti non rispondenti al vero ovvero omettono fatti materiali rilevanti la cui comunicazione è imposta dalla legge sulla situazione economica, patrimoniale o finanziaria della società o del gruppo al quale la stessa appartiene, in modo concretamente idoneo ad indurre altri in errore, sono puniti con la pena della reclusione da uno a cinque anni.

La stessa pena si applica anche se le falsità o le omissioni riguardano beni posseduti o amministrati dalla società per conto di terzi».

Articolo n. 10 - Introduzione degli articoli 2621-bis e 2621ter del codice civile.

1. Dopo l'articolo 2621 del codice civile sono inseriti i seguenti:

«Art. 2621-bis (Fatti di lieve entità). - Salvo che costituiscano più grave reato, si applica la pena da sei mesi a tre anni di reclusione se i fatti di cui all'articolo 2621 sono di lieve entità, tenuto conto della natura e delle dimensioni della società e delle modalità o degli effetti della condotta. Salvo che costituiscano più grave reato, si applica la stessa pena di cui al comma precedente quando i fatti di cui all'articolo 2621 riguardano società che non superano i limiti indicati dal secondo comma dell'articolo 1 del regio decreto 16 marzo 1942, n. 267. In tale caso, il delitto è procedibile a querela della società, dei soci, dei creditori o degli altri destinatari della comunicazione sociale.

Art. 2621-ter (Non punibilità per particolare tenuità). - Ai fini della non punibilità per particolare tenuità del fatto, di cui all'articolo 131-bis del codice penale, il giudice valuta, in modo prevalente, l'entità dell'eventuale danno cagionato alla società, ai soci o ai creditori conseguente ai fatti di cui agli articoli 2621 e 2621-bis».

Articolo n. 11 - Modifica dell'articolo 2622 del codice civile. 1. L'articolo 2622 del codice civile è sostituito dal seguente: «Art. 2622 (False comunicazioni sociali delle società quotate). - Gli amministratori, i direttori generali, i dirigenti preposti alla redazione dei documenti contabili societari, i sindaci e i liquidatori di società emittenti strumenti finanziari ammessi alla negoziazione in un mercato regolamentato italiano o di altro Paese dell'Unione europea, i quali, al fine di conseguire per sé o per altri un ingiusto profitto, nei bilanci, nelle relazioni o nelle altre comunicazioni sociali dirette ai soci o al pubblico consapevolmente espongono fatti materiali non rispondenti al vero ovvero omettono fatti materiali rilevanti la cui comunicazione è imposta dalla legge sulla situazione economica, patrimoniale o finanziaria della società o del gruppo al quale la stessa appartiene, in modo concretamente idoneo ad indurre altri in errore, sono puniti con la pena della reclusione da tre a otto anni.

Alle società indicate nel comma precedente sono equiparate:

1) le società emittenti strumenti finanziari per i quali è stata presentata una richiesta di ammissione alla negoziazione in un mercato regolamentato italiano o di altro Paese dell'Unione europea;

2) le società emittenti strumenti finanziari ammessi alla negoziazione in un sistema multilaterale di negoziazione italiano;

3) le società che controllano società emittenti strumenti finanziari ammessi alla negoziazione in un mercato regolamentato italiano o di altro Paese dell'Unione europea; 4) le società che fanno appello al pubblico risparmio o che comunque lo gestiscono. 
Le disposizioni di cui ai commi precedenti si applicano anche se le falsità o le omissioni riguardano beni posseduti $\mathrm{o}$ amministrati dalla società per conto di terzi».

Articolo n. 12 - Modifiche alle disposizioni sulla responsabilità amministrativa degli enti in relazione ai reati societari.

1. All'articolo 25-ter, comma 1 , del decreto legislativo 8 giugno 2001, n. 231, sono apportate le seguenti modificazioni:

a) l'alinea è sostituito dal seguente: «In relazione ai reati in materia societaria previsti dal codice civile, si applicano all'ente le seguenti sanzioni pecuniarie:»;

b) la lettera a) è sostituita dalla seguente:

«a) per il delitto di false comunicazioni sociali previsto dall'articolo 2621 del codice civile, la sanzione pecuniaria da duecento a quattrocento quote»;

c) dopo la lettera a) è inserita la seguente:

«a-bis) per il delitto di false comunicazioni sociali previsto dall'articolo 2621-bis del codice civile, la sanzione pecuniaria da cento a duecento quote»;

d) la lettera b) è sostituita dalla seguente:

«b) per il delitto di false comunicazioni sociali previsto dall'articolo 2622 del codice civile, la sanzione pecuniaria da quattrocento a seicento quote»;

e) la lettera c) è abrogata".

\section{Il testo previgente - testo introdotto dal D.Lgs. 11 aprile 2002 n. 61.}

“Articolo n. N.1 - Nuove disposizioni sugli illeciti penali ed amministrativi in materia di società e di consorzi.

1. Il Titolo XI del libro V del codice civile è sostituito dal seguente:

Titolo XI - Disposizioni Penali in Materia di Società e di Consorzi.

Capo I - Delle falsità.

Articolo 2621 - False comunicazioni sociali.

Salvo quanto previsto dall'articolo 2622, gli amministratori, i direttori generali, i sindaci e i liquidatori, i quali, con l'intenzione di ingannare $\mathrm{i}$ soci o il pubblico e al fine di conseguire per sé o per altri un ingiusto profitto, nei bilanci, nelle relazioni o nelle altre comunicazioni sociali previste dalla legge, dirette ai soci o al pubblico, espongono fatti materiali non rispondenti al vero ancorché oggetto di valutazioni ovvero omettono informazioni la cui comunicazione è imposta dalla legge sulla situazione economica, patrimoniale, o finanziaria della società o del gruppo al quale essa appartiene, in modo idoneo ad indurre in errore i destinatari sulla predetta situazione, sono puniti con l'arresto fino ad un anno e sei mesi.

La punibilità è estesa anche al caso in cui le informazioni riguardino beni posseduti od amministrati dalla società per conto di terzi.

La punibilità è esclusa se le falsità o le omissioni non alterano in modo sensibile la rappresentazione della situazione economica, patrimoniale o finanziaria della società o del gruppo al quale essa appartiene. La punibilità è comunque esclusa se le falsità o le omissioni determinano una variazione del risultato economico di esercizio, al lordo delle imposte, non superiore al $5 \%$ o una variazione del patrimonio netto non superiore all'1 per cento.

In ogni caso il fatto non è punibile se conseguenza di valutazioni estimative che, singolarmente considerate, differiscono in misura non superiore al 10 per cento da quella corretta.

Articolo 2622 - False comunicazioni sociali in danno dei soci o dei creditori.

Gli amministratori, i direttori generali, i sindaci e i liquidatori, i quali, con l'intenzione di ingannare i soci o il pubblico e al fine di conseguire per sé o per altri un ingiusto profitto, nei bilanci, nelle relazioni o nelle altre comunicazioni sociali previste dalla legge, dirette ai soci o al pubblico, esponendo fatti materiali non rispondenti al vero ancorché oggetto di valutazioni, ovvero omettendo informazioni la cui comunicazione è imposta dalla legge sulla situazione economica, patrimoniale o finanziaria della società o del gruppo al quale essa appartiene, in modo idoneo ad indurre in errore $\mathrm{i}$ destinatari sulla predetta situazione, cagionano un danno patrimoniale ai soci o ai creditori sono puniti, a querela della persona offesa, con la reclusione da sei mesi a tre anni.

Si procede a querela anche se il fatto integra altro delitto, ancorché aggravato a danno del patrimonio di soggetti diversi dai soci e dai creditori, salvo che sia commesso in danno dello Stato, di altri enti pubblici o delle Comunità europee.

Nel caso di società soggette alle disposizioni della parte IV, titolo III, capo II, del decreto legislativo 24 febbraio 1998, n. 58, la pena per i fatti previsti al primo comma è da uno a quattro anni e il delitto è procedibile d'ufficio. 
La punibilità per i fatti previsti dal primo e terzo comma è estesa anche al caso in cui le informazioni riguardino beni posseduti o amministrati dalla società per conto di terzi.

La punibilità per i fatti previsti dal primo e terzo comma è esclusa se le falsità o le omissioni non alterano in modo sensibile la rappresentazione della situazione economica, patrimoniale o finanziaria della società o del gruppo al quale essa appartiene. La punibilità è comunque esclusa se le falsità o le omissioni determinano una variazione del risultato economico di esercizio, al lordo delle imposte, non superiore al 5 per cento o una variazione del patrimonio netto non superiore all'1 per cento.

In ogni caso il fatto non è punibile se conseguenza di valutazioni estimative che, singolarmente considerate, differiscono in misura non superiore al 10 per cento da quella corretta”.

\section{L'esame della nuova legge menzionata -} Elementi costitutivi del reato.

Poste le discipline in raffronto, sopra riportate per esteso, può ora passarsi all'esame della nuova disciplina.

"La legge menzionata ha infatti configurato due autonomi titoli di reato, configurati entrambi come delitti e collocati, rispettivamente, nei citati artt. 2621 e 2622 c.c. al fine di differenziare la repressione delle false comunicazioni sociali a seconda che il fatto sia commesso nell'ambito di una società 'non quotata' ovvero di una 'quotata'. Differenziazione che si traduce soprattutto nella previsione di diverse cornici edittali di pena: $\underline{\text { da uno }}$ a cinque anni di reclusione nel primo caso, da tre a otto nel secondo. Ed infatti, a parte alcuni pur non marginali dettagli di cui si dirà in seguito, la struttura delle due incriminazioni è pressochè identica e tesa a superare l'assetto ideato dal legislatore del 2002 nel quale era prevista una fattispecie contravvenzionale di pericolo ed un delitto di danno - in un rapporto di sostanziale progressione criminosa tra loro - quest'ultimo diversamente configurato qualora il fatto riguardasse una quotata esclusivamente in merito al profilo del trattamento sanzionatorio ed al regime di procedibilità.

La novella propone invece due reati di pericolo (invero tre se si considera anche l'ipotesi attenuata di cui all'art. 2621-bis c.c., configurata come vero e proprio titolo autonomo di reato), integrati a prescindere dalla causazione di un danno a soci o creditori, che ripropongono in buona parte il profilo strutturale della fattispecie contravvenzionale contenuta nel previgente testo dell'art. 2621 c.c.

Scompare altresi per le società non quotate la procedibilità a querela della persona offesa, rivelandosi in tal senso l'intenzione di recuperare coerenza sistematica attraverso la tutela esclusiva della trasparenza dell'informazione societaria. Quelli di nuovo conio rimangono invece reati propri degli amministratori, dei direttori generali, dei dirigenti preposti alla redazione dei documenti contabili societari, dei sindaci e dei liquidatori.

Con riguardo all'oggetto materiale del reato è stata conservata la tipizzazione delle comunicazioni sociali rilevanti introdotta dalla precedente riforma del 2002, individuate nei bilanci, nelle relaz̧ioni e nelle altre comunicazioni dirette ai soci e al pubblico previste dalla legge. Invero tale ultimo inciso è stato 'spostato', rispetto alla formulazione previgente, in coda all'elenco, con l'apparente intento di fugare eventuali residui dubbi circa il fatto che la specificazione riguardi non solo le comunicazioni, ma altresi le relazioni. Viene dunque confermata l'irrilevanza penale delle condotte che riguardano comunicazioni 'atipiche', comunicazioni interorganiche e quelle dirette ad unico destinatario, sia esso un soggetto privato o pubblico, le quali, sussistendone le condizioni, possono configurare, a seconda dei casi, $i$ reati di truffa ovvero quelli previsti dagli artt. 2625, 2637 e 2638 c.c. o ancora quello di cui all'art. 185 TUIF.

Il legislatore ha invece provveduto, come già accennato, all'eliminazione dell'evento di danno e delle soglie previsti 
nella previgente formulazione dei due articoli menzionati. Eliminazione cui ha corrisposto una rimodulazione delle condotte tipiche, ora integrate dall'esposizione in una delle comunicazioni tipizzate di fatti materiali non rispondenti al vero' ovvero nell'omissione di 'fatti materiali la cui comunicazione è imposta dalla legge sulla situazione economica, patrimoniale o finanziaria della società o del gruppo al quale la stessa appartiene'. Nell'ipotesi prevista dall'art. 2621 - dedicata come ricordato alle sole società non quotate - $i$ 'fatti materiali' non rispondenti al vero ovvero quelli occultati devono inoltre essere 'rilevanti'.

La novella ha dunque ripreso la molto discussa formula utilizzata dal legislatore del 2002 per circoscrivere l'oggetto della condotta attiva, amputandola però del riferimento alle valutaẓioni ('ancorchè oggetto di valutazioni' contenuto nel testo previgente dei due articoli e provvedendo contestualmente a replicarla anche nella definizione di quello della condotta omissiva, in relazione alla quale le due norme incriminatrici in precedenza evocavano le 'informazioni' oggetto di omessa comunicazione.

Sempre con riguardo all'elemento oggettivo delle due fattispecie, ̀̀ stato inoltre riproposto il requisito dell'idoneità ingannatoria della falsa comunicazione (e cioè della attitudine delle medesime ad indurre in errore $i$ loro destinatari), che ̀̀ stato peraltro 'rafforzato' attraverso l'aggiunta dell'avverbio 'concretamente', in grado di qualificare $i$ due delitti come reati di pericolo, per l'appunto, concreto.

Per quanto riguarda invece le modifiche apportate alla struttura dell'elemento soggettivo, deve osservarsi come il legislatore abbia confermato, con riguardo ad entrambe le figure di reato, la necessità di un dolo specifico, caratterizzato dal fine di procurare per sé o per altri un ingiusto profitto. La novella non ha invece riproposto la espressa caratterizzazione dello stesso come intenzionale, attraverso la soppressione dell'inciso 'con l'intenzione di ingannare $i$ soci o il pubblico' che era stato introdotto nel 2002. In diretta relazione alla descrizione della condotta ha fatto invece la sua comparsa l'avverbio 'consapevolmente', che appare sintomatico della volontà del legislatore di escludere la rilevanza del dolo eventuale".

E dunque:

a) La novella ha introdotto due autonomi titoli di reato, entrambi configurati come delitto (artt. 2621 e 2622 cod. civ. al fine di differenziare la repressione delle false comunicazioni sociali a seconda che il fatto sia commesso nell'ambito di una società "non quotata" ovvero di una società "quotata"); scompare l'ipotesi contravvenzionale di cui al previgente art. 2621 cod. civ.;

b) Differenziazione che si è tradotta nella previsione di diverse forbici edittali (art. 2621 cod. civ. da uno a cinque anni; art. 2622 cod. civ. da tre a otto anni; art. 2621 bis cod. civ. da sei mesi a tre anni di reclusione; è stata introdotta una causa di non punibilità per particolare tenuità del fatto, ai sensi dell'art. 2621ter cod. civ. ${ }^{2}$ );

\footnotetext{
${ }^{1}$ Cfr. Cassazione penale sez. V - 8/7/2015 (ud. 8/7/2015, dep.16/9/2015 ) - N. 37570 - Pres. Marasca - Rel. Pistorelli.

${ }^{2}$ Cfr. Relazione per la Quinta Sezione Penale (Riunione sezionale del 15 ottobre 2015) - Rel.: V/003/15 Redattore: Silvestri - Paragrafo 1. La riformulazione della false comunicazioni sociali. Cenni: "Il nuovo assetto dei reati di false comunicazioni sociali a seguito entrata in vigore della 1. 27 maggio 2015, n. 69, è costituito da due fattispecie incriminatrici (artt. $2621 \mathrm{e}$ 2622), caratterizzate entrambe come reati di pericolo e differenziate alla luce della tipologia societaria, e da due norme (artt. 2621 bis e 2621 ter) riferite solo all' art. 2621 e contenenti una cornice di pena più mite per i fatti di "lieve entità" e una causa di non punibilità per la loro "particolare tenuità". È stata confermata l'architettura a "piramide punitiva" degli illeciti in materia di false comunicazioni sociali, ma la struttura dell'impianto è fondata da soli delitti, essendosi abbandonato il modello
} 
c) Vengono proposti due reati di pericolo (anzi tre considerata l'ipotesi attenuata ex art. 2621 - bis cod. civ. che costituisce autonomo titolo di reato);

d) Tali delitti vengono integrati a prescindere dalla causazione di un danno ai soci o ai creditori;

e) Scompare per le società non quotate la procedibilità a querela della persona offesa;

f) Mantengono la struttura di reati propri degli amministratori, dei direttori generali, dei dirigenti preposti alla redazione dei documenti contabili societari, dei sindaci e dei liquidatori;

g) La nuova disciplina ha eliminato l'evento di danno e le soglie previste nella previgente formulazione; viene circoscritto "l'oggetto della condotta attiva, amputandola però del riferimento alle valutazioni ('ancorchè oggetto di valutarioni') contenuto nel testo previgente dei due articoli e provvedendo contestualmente a replicarla anche nella definizione di quello della condotta

contravvenzionale che caratterizzava la previgente incriminazione contenuta nell'art. $2621 \mathrm{cod}$. civ. per le aziende non quotate in Borsa, nonché l'illecito amministrativo introdotto nel 2005 all'interno delle figure in questione (1. n. 262 del 2005). Al livello più basso della piramide si pongono le due meno gravi figure delittuose dei fatti di lieve entità (art. 2621-bis cod. civ.), la cui cornice edittale è da sei mesi a tre anni di reclusione; per la seconda ipotesi di lieve entità, basata sul concetto di imprenditore commerciale non fallibile, è altresì stabilita la procedibilità a querela della società, soci e altri destinatari della comunicazione sociale. Al gradino intermedio è stato collocato il delitto di false comunicazioni sociali di cui all'art. 2621 cod. civ. Al vertice della piramide è posto l'art. $2622 \mathrm{cod}$. civ., con riferimento alle società quotate in Italia o in altri mercati regolamentati dell'Unione Europea (l'art. 2622, comma 2 , cod. civ. equipara alle citate società quotate altre tipologie: le società che hanno fatto richiesta di ammissione alla Borsa, le società che emettono strumenti finanziari in un sistema multilaterale di negoziazione, le società controllanti e quelle che fanno appello al pubblico risparmio o lo gestiscono).

omissiva, in relazione alla quale le due norme incriminatrici in precedenza evocavano le 'informazioni' oggetto di omessa comunicazione" "3; -in argomento si tornerà;

h) Quanto all'elemento soggettivo, il legislatore ha confermato la necessità di un dolo specifico per entrambe le fattispecie di reato di cui agli artt. 2621, 2622 cod. civ. ("al fine di conseguire per sè o per altri un ingiusto profitto" - si è mantenuta la medesima formula di cui alla disciplina previgente); l'aggiunta dell'avverbio "consapevolmente" porta ad escludere la rilevanza del dolo eventuale.

4. L'interpretazione giurisprudenza successorio - art. 2 cod. pen. - tra la previgente disciplina e quella riformatrice -; primi problemi interpretativi - riassunto della Sentenza “Crespi”.

$\mathrm{Si}$ evidenzia in generale come "le modifiche apportate dalla L. n. 69 del 2015 abbiano innanzi $i$ tutto ampliato l'ambito di operatività dell'incriminazione delle false comunicazioni sociali, avendo comportato, come evidenziato, l'eliminazione dell'evento e delle soglie previste dal precedente testo dell'art. 2622 c.c., mantenendo invece nella sostanza identico il profilo della condotta tipica. In tal senso l'odierno fenomeno successorio assume caratteristiche opposte a quello generato dal D.Lgs. n. 61 del 2002, che aveva invece ristretto gli orizzonti applicativi della fattispecie tracciati nell'originario

\footnotetext{
${ }^{3}$ Cfr. ancora Cfr. Cassazione penale sez. V - 8/7/2015 (ud. 8/7/2015, dep.16/9/2015) - N. 37570 - Pres. Marasca - Rel. Pistorelli.
} 
testo della disposizione del codice civile. Ma non è in dubbio che tra la fattispecie previgente e quella di nuova configurazione nell'art. 2621 c.c. sussista un evidente rapporto di continuità normativa"4.

Appena prima la medesima sezione aveva espresso la medesima opinione, ripresa dalla sentenza sopra citata: "Deve allora osservarsi come le modifiche apportate dalla L. n. 69 del 2015 abbiano innanzi tutto ampliato l'ambito di operatività dell'incriminazione delle false comunicazioni sociali, avendo comportato, come evidenziato, l'eliminazione dell'evento e delle soglie previste dal precedente testo dell'art. 2622 cod. civ., mantenendo invece parzialmente coincidente il profilo della condotta tipica. In tal senso l'odierno fenomeno successorio finisce per assumere caratteristiche opposte a quello generato dal D.Lgs. n. 61 del 2002, che aveva invece ristretto gli ambiti applicativi della fattispecie tracciati nell'originario testo della disposizione del codice civile" 5 .

"Ovviamente le evidenziate differenze nella modulazione della fattispecie, se non determinano discontinuità, evidenżiano però la necessità ai sensi dell'art. 2 c.p., comma 4 di stabilire quale sia la norma più favorevole. Valutazione che deve essere operata in concreto, comparando le diverse discipline sostanziali succedutesi nel tempo nel loro complesso. Ed in tal senso nel caso di specie la norma più favorevole appare essere quella di cui all'art. 2622 c.c. nella sua previgente formulazione in ragione della maggiore selettività della fattispecie tipizzata"6. In linea generale non paiono dunque profilarsi particolari questioni di

\footnotetext{
${ }^{4}$ Ibidem.

5 Cfr. Cassazione penale sez. V - 16/6/2015 (ud. 16/6/2015, dep.30/7/2015 ) - N. 33774 - Pres. Lombardi - Rel. Miccoli - pag. 54.

6 Ancora Cassazione penale sez. V - 8/7/2015 (ud. 8/7/2015, dep.16/9/2015) - N. 37570 - Pres. Marasca Rel. Pistorelli.
}

discontinuità normativa tra vecchia e nuova disciplina, come invece avvenuto a seguito della novella del 2002 introdotta dal D.Lgs. 11 aprile 2002 n. 617, salvo per quanto riguarda la cennata questione correlata alla descrizione, da parte del legislatore del 2015, della condotta del reato di false comunicazioni sociali in termini di esposizione di "fatti materiali rilevanti non rispondenti al vero", ove non figura più il riferimento alle valutazioni ("ancorché oggetto di valutazioni") contenuto nel testo previgente dei due articoli, laddove, con riguardo all'ipotesi omissiva, si è provveduto alla sostituzione del termine "informazioni" con la locuzione "fatti materiali".

\footnotetext{
${ }^{7}$ La fattispecie prevista dall'art. 223, comma 2, n. $1 \mathrm{~L}$. Fall. è reato di evento (costituito dal dissesto) ma a condotta vincolata, stante il richiamo espresso operato dalla norma alla commissione di "alcuno dei fatti previsti dagli articoli 2621, 2622, 2626, 2627, 2628, 2629, 2632, 2633 e 2634 del codice civile". In tale contesto, la novella del 2002 aveva introdotto modificazioni significative quanto alle ipotesi di bancarotta impropria da reato societario di cui al comma 2 dell'art. 223, comma 2, n. 1 L. Fall., facendo concludere in un primo momento, al riguardo, la giurisprudenza di legittimità per la tesi della abolitio criminis (così Cassazione penale sez. V - 8 ottobre 2002 n. 34622 - che aveva affermato che la nuova formulazione dell'art. 223 1. fall. (fatti di bancarotta fraudolenta commessi da amministratori o sindaci di società fallite) ha comportato non già una mera successione di leggi penali nel tempo, ma una vera e propria "abolitio criminis" della vecchia fattispecie incriminatrice. Con la conseguenza che, anche se è intervenuta sentenza di condanna, il reo può chiedere ex art. 673 c.p.p. la revoca della sentenza). Sono poi intervenute le Sezioni Unite che hanno precisato essere l'abolitio criminis solo parziale (Sez. U., sentenza del 26/3/2003 n. 25887 Ud. - dep. 16/6/2003 - Riv. 224605 Giordano e altri che ha stabilito come la nuova formulazione delle norme che prevedono i delitti di false comunicazioni sociali e di bancarotta fraudolenta impropria "da reato societario" ad opera, rispettivamente, degli articoli 1 e 4 del decreto legislativo 11 aprile 2002, n. 61 non ha comportato l'abolizione totale dei reati precedentemente contemplati, ma ha determinato una successione di leggi con effetto parzialmente abrogativo in relazione a quei fatti, commessi prima dell'entrata in vigore del citato decreto, che non siano riconducibili alle nuove fattispecie criminose).
} 
Tale questione, già pervenuta all'esame della Corte di Cassazione, rende dunque necessario verificare se nel passaggio alla recente riforma - la quale come detto nel tipizzare la condotta (sia attiva che omissiva) mutua solo la locuzione "fatti materiali", senza riferimento alcuno alle valutazioni (nella versione previgente si faceva riferimento espresso a valutazioni ("ancorché oggetto di valutazioni"), possa legittimare una interpretazione abrogans che esclude penale rilevanza ai fatti derivanti da procedimento valutativo.

In altri termini, se è possibile accedere ad una interpretazione restrittiva della nozione di "fatti materiali" muovendo dall'esame della formulazione della nuova fattispecie.

La più volte citata Cassazione penale sez. V - 8/7/2015 (ud. 8/7/2015, dep.16/9/2015), n. 37570, Pres. Marasca, Rel. Pistorelli affronta solo incidentalmente tale questione, poiché in quel caso la contestazione mossa all'imputato "riguarda la mancata esposizione nel bilancio di poste attive effettivamente esistenti nel patrimonio della società. Un fatto, dunque, riconducibile allo schema della nuova incriminazione anche qualora dovesse propendersi per una interpretazione restrittiva della nozione di 'fatti materiali"”.

Tuttavia i giudici di legittimità, nella premessa che "non è in dubbio che tra la fattispecie previgente e quella di nuova configurazione nell'art. 2621 c.c. sussista un evidente rapporto di continuità normativa", affermano, sulla questione specifica: "Qualche perplessità può suscitare la già segnalata epurazione dello specifico riferimento alle valutazioni contenuto nel testo previgente dei due articoli e alla sostituzione, con riguardo allipotesi omissiva, del termine informazioni' con la locuzione 'fatti materiali'. Scelte che se dovessero essere interpretate nel senso di escludere la rilevanza del falso cd. 'qualitativo' indubbiamente determinerebbero, al contrario, un ridimensionamento dell'elemento oggettivo delle false comunicazioni sociali. Ma si tratterebbe in parte qua di un effetto solo parzialmente abrogativo, limitato a quei fatti che non troverebbero più corrispondenza nelle nuove previsioni normative. Si tratta comunque di questione che non necessita di essere approfondita in questa sede, atteso che l'oggetto della contestazione mossa all'imputato riguarda la mancata esposizione nel bilancio di poste attive effettivamente esistenti nel patrimonio della società. Un fatto, dunque, riconducibile allo schema della nuova incriminazione anche qualora dovesse propendersi per una interpretazione restrittiva della nozione di 'fatti materiali".

La questione è stata invece affrontata funditus dalla citata Cassazione penale sez. V 16/6/2015 (ud. 16/6/2015, dep. 30/7/2015), n. 33774, Pres. Lombardi, Rel. Miccoli - cd. sentenza Crespi (Crespi noto sondaggista di Berlusconi) - che è giunta, tenuto conto della nuova formulazione dell'art. 2621 cod. civ. (i cui fatti sono richiamati dall'art. 223, comma 2, n. 1 1. fall. così come ascritti all'imputato - capo 19 della rubrica), ad annullare la sentenza, senza rinvio, in ordine all'imputazione di bancarotta impropria da reato societario, ritenendo applicabile al caso in esame la disciplina di cui all'art. 2 cod. pen. perché fatto non più previsto dalla legge come reato, atteso che le condotte ascritte erano tutte incentrate sul mancato controllo dei valori estimativi. 
Questo lo snodo argomentativo che ha portato i giudici di legittimità ad una interpretazione restrittiva della nozione di "fatti materiali"s:

a) Il legislatore della riforma del 2002 aveva utilizzato la locuzione "fatti materiali non rispondenti al vero", precisando che oggetto dei "fatti materiali" potessero essere anche le valutazioni, sostanzialmente recependo la consolidata interpretazione giurisprudenziale e dottrinaria del termine "fatti" contenuto nell'originaria formulazione dell'art. 2621.

Espresso riferimento alle "valutazioni estimative" poi si era fatto prevedendo le soglie di punibilità di cui all'art. 2621, comma 4 e all'art. 2622, comma 89.

\footnotetext{
8 Cfr. Cassazione penale sez. V - 16/6/2015 (ud. 16/6/2015, dep. 30/7/2015) - N. 33774 - Pres. Lombardi - Rel. Miccoli: "Nell'analisi ermeneutica da compiersi, si ribadisce in primo luogo che la riforma del 2015 ha ripreso la formula utilizzata dal legislatore del 2002 per circoscrivere l'oggetto della condotta attiva, privandola però del riferimento alle valutazioni e provvedendo contestualmente a replicarla anche nella definizione di quello della condotta omissiva, in relazione alla quale il testo previgente faceva invece riferimento alle 'informazioni'. Tutto ciò non può essere ritenuto neutro nella interpretazione delle norme in esame, avuto riguardo in primo luogo all'esigenza di tipizzazione della condotta e alla corretta applicazione dei criteri ermeneutici in materia penale. Di rilievo è evidenziare, per quanto si desume dall'iter dei lavori parlamentari, come il testo delle norme approvato sia il frutto di uno specifico emendamento, che ha sostituito quello inizialmente presentato (risultante dall'unificazione dei vari disegni di legge), il quale, nel descrivere l'oggetto tanto della condotta commissiva che di quella omissiva, aveva utilizzato il termine 'informazioni'”.

9 Autorevole dottrina aveva evidenziato che "..alla luce della riforma della fattispecie di false comunicazioni sociali sembra potersi affermare che il criterio del vero legale abbia certamente perso di attualità. Infatti, l'aver previsto all'art. 2621, comma 4 (e all'art. 2622, comma 6) la non punibilità del fatto per le "valutazioni estimative che, singolarmente considerate, differiscono in misura non superiore al $10 \%$ da quella corretta' smentisce ogni possibilità di far coincidere la falsità penalmente rilevante con la violazione sic et simpliciter dei criteri civilistici per la formazione del bilancio e per la valutazione delle
}

Dunque, ad avviso della Corte, l'adozione dello stesso riferimento ai "fatti materiali non rispondenti al vero", senza alcun richiamo alle valutazioni e il dispiegamento della formula citata anche nell'ambito della descrizione della condotta omissiva consentirebbe di ritenere ridotto l'ambito di operatività delle due nuove fattispecie di false comunicazioni sociali, con esclusione dei cosiddetti falsi valutativi.

Tanto più che i testi riformati degli artt. 2621 e 2622 si inseriscono in un contesto normativo che vede ancora un esplicito riferimento alle valutazioni nell'art. 2638 cod. civ. ${ }^{10}$ (Ostacolo all'esercizio delle funzioni delle autorità pubbliche di vigilanza), peraltro proprio

singole poste" - così: Musco E., I nuovi reati societari, Giuffrè, Milano, 2007.

10 Articolo n. 2638 Cod. Civ. - Ostacolo all'esercizio delle funzioni delle autorità pubbliche di vigilanza.

[I]. Gli amministratori, i direttori generali, i dirigenti preposti alla redazione dei documenti contabili societari, $i$ sindaci e i liquidatori di società o enti e gli altri soggetti sottoposti per legge alle autorità pubbliche di vigilanza, o tenuti ad obblighi nei loro confronti, i quali nelle comunicazioni alle predette autorità previste in base alla legge, al fine di ostacolare l'esercizio delle funzioni di vigilanza, espongono fatti materiali non rispondenti al vero, ancorché oggetto di valutazioni, sulla situazione economica, patrimoniale o finanziaria dei sottoposti alla vigilanza ovvero, allo stesso fine, occultano con altri mezzi fraudolenti, in tutto o in parte fatti che avrebbero dovuto comunicare, concernenti la situazione medesima, sono puniti con la reclusione da uno a quattro anni. La punibilità è estesa anche al caso in cui le informazioni riguardino beni posseduti o amministrati dalla società per conto di terzi. [II]. Sono puniti con la stessa pena gli amministratori, i direttori generali, i dirigenti preposti alla redazione dei documenti contabili societari, i sindaci e i liquidatori di società, o enti e gli altri soggetti sottoposti per legge alle autorità pubbliche di vigilanza o tenuti ad obblighi nei loro confronti, i quali, in qualsiasi forma, anche omettendo le comunicazioni dovute alle predette autorità, consapevolmente ne ostacolano le funzioni. [III]. La pena è raddoppiata se si tratta di società con titoli quotati in mercati regolamentati italiani o di altri Stati dell'Unione europea o diffusi tra il pubblico in misura rilevante ai sensi dell'articolo 116 del testo unico di cui al decreto legislativo 24 febbraio 1998, n. 58. 
a precisazione contenutistica della stessa locuzione "fatti materiali non rispondenti al vero". Osservano che tale disposizione (art. 2638 cod. civ.) continua a punire $\mathrm{i}$ medesimi soggetti attivi ("gli amministratori, i direttori generali, i dirigenti preposti alla redazione dei documenti contabili societari, i sindaci e i liquidatori di società....") dei reati di cui agli artt. 2621 e 2622 che, nelle comunicazioni dirette alle autorità pubbliche di vigilanza, "espongono fatti materiali non rispondenti al vero, ancorchè oggetto di valutazioni". In tal senso fanno riferimento ad una necessaria lettura delle norme ancorata al canone interpretativo ubi lex voluit dixit, ubi noluit tacuit affermando di non poter trascurare la circostanza dell'inserimento di modifiche normative in un sistema che riguarda la rilevanza penale delle attività societarie con una non giustificata differenziazione dell'estensione della condotta tipizzata in paralleli ambiti operativi, quali sono quelli degli artt. 2621 e 2622 cod. civ., da una parte, e art. 2638 cod. civ., dall'altra, norme che, sebbene tutelino beni giuridici diversi, sono tutte finalizzate a sanzionare la frode nell'adempimento dei doveri informativi.

In tal senso, in argomento, richiamano l'orientamento di legittimità laddove, in particolare, si è affermato che "in tema di false comunicazioni all'autorità di vigilanza, premesso che esiste continuità normativa tra la fattispecie prevista dal
D.Lgs. n. 385 del 1993, abrogato art. 134 e quella prevista dall'art. 2638 cod. civ., quale novellato dal D.Lgs. n. $61 \mathrm{del}$ 2002, deve ritenersi tuttora configurabile il reato anche nel caso in cui la falsità sia contenuta in giudizi estimativi delle poste di bilancio, atteso che dal novero dei 'fatti materiali', indicati dall'attuale norma incriminatrice come possibile oggetto della falsità, vanno escluse soltanto le previsioni o congetture prospettate come tali, vale a dire quali apprezzamenti di carattere squisitamente soggettivo, e l'espressione, riferita agli stessi fatti, 'ancorché soggetti a valutazione', va intesa in senso concessivo, per cui, in ultima analisi, l'oggetto della vigente norma incriminatrice viene a corrispondere a quello della precedente, che prevedeva come reato la comunicazione all'autorità di vigilanza di 'fatti non rispondenti al vero"' (Sez. 5, 28/9/2005 n. 44702, P.G. in proc. Mangiapane ed altri, Rv. 232535). Tanto si è detto, però, nell'ambito di un giudizio nel quale erroneamente la sentenza impugnata aveva ritenuto che con l'inciso "ancorché oggetto di valutazioni" si volesse escludere la rilevanza penale delle false valutazioni. Questa Corte ha avuto quindi la necessità di osservare che, "benché la locuzione abbia dato luogo in dottrina a varie dispute, va considerato che la congiunzione 'ancorché' - equivalente a 'sebbene, quantunque, anche se' - ha, nel contesto generale della norma, valore concessivo. 
Pertanto, nell'impostazione della nuova fattispecie la proposizione 'fatti materiali non rispondenti al vero, ancorché oggetto di valutazione' finisce col corrispondere in tutto all'espressione, contenuta nella disposizione del D.Lgs. n. 385 del 1993, abrogato art. 134 'fatti non rispondenti al vero'. Espressione, si sottolinea, del tutto corrispondente a quella contenuta nell'art. 2621 cod. civ. previgente alla riforma del 2002. Né può trascurarsi che la giurisprudenza di questa stessa Sezione ha ritenuto necessario precisare che non deve trarre in inganno l'utilizzo, all'interno dell'art. 2638 cod. civ., comma 1 dell'espressione 'fatti materiali', proprio perché la quasi immediata aggiunta 'ancorché oggetto di valutazioni' vale ad estendere la portata della previsione, escludendone soltanto le previsioni o congetture prospettate come tali (si veda in motivazione Sez. 5, 7/12/2012 n. 49362, Pg in proc. Banco e altri, Rv. 254065). Tale interpretazione della locuzione contenuta nell'art. 2638 cod. civ. allora finisce per ulteriormente supportare la tesi secondo la quale la riforma del 2015 ha fatto ricorso solo alla locuzione 'fatti materiali non rispondenti al vero', non replicando la precisazione contenuta del previgente art. 2621 e nel vigente citato art. 2638, con l'intento di lasciar fuori dalla fattispecie le valutazioni” (così a pag. 5658 sentenza).

b) Dunque "Tutto ciò non può essere ritenuto neutro nella interpretazione delle norme in esame, avuto riguardo in primo luogo all'esigenza di tipizzazione della condotta e alla corretta applicazione dei criteri ermeneutici in materia penale. Di rilievo è evidenziare, per quanto si desume dall'iter dei lavori parlamentari, come il testo delle norme approvato sia il frutto di uno specifico emendamento, che ha sostituito quello inizialmente presentato (risultante dall'unificazione dei vari disegni di legge), il quale, nel descrivere l'oggetto tanto della condotta commissiva che di quella omissiva, aveva utilizzato il termine 'informazioni'. In particolare, il testo dell'art. 2621 cod. civ. sottoposto inizialmente all'esame della competente commissione prevedeva la seguente condotta '...consapevolmente espongono informazioni non rispondenti al vero ovvero omettono informazioni la cui comunicazione è imposta dalla legge sulla situazione economica, patrimoniale o finanziaria della società o del gruppo al quale la stessa appartiene, in modo concretamente idoneo ad indurre altri in errore, sono puniti con la pena della reclusione da uno a cinque anni..... I primi commenti di una parte della dottrina sulla nuova disciplina hanno rilevato immediatamente come il riferimento introdotto dall'emendamento all'omissione di fatti materiali anziché di informazioni, unitamente alla mancata riproposizione dell'inciso 'ancorché oggetto di valutazioni' in relazione alla esposizione di fatti materiali non rispondenti al vero, 
finisse per essere letta come la volontà di non attribuire più rilevanza penale alle attività di mera valutazione ovvero alle stime, che caratterizzano alcune voci di bilancio e che rispondono a una pluralità di considerazioni fondate su elementi di varia natura. E si è pure rilevato che la locuzione 'fatti materiali', senza ulteriori specificazioni, già era stata utilizzata dalla L. n. 154 del 1991 per circoscrivere l'oggetto del reato di frode fiscale di cui alla L. n. 516 del 1982, art. 4, lett. f), con il chiaro intento di escludere dall'incriminazione le valutazioni relative alle componenti attive e passive del reddito dichiarato. Il citato art. 4 , lett. f), puniva infatti l'utilizzazione di 'documenti attestanti fatti materiali non corrispondenti al vero', nonché il compimento di 'comportamenti fraudolenti idonei ad ostacolare l'accertamento di fatti materiali'. Pacificamente una tale formulazione del dato normativo comportava l'irrilevanza penale di qualsiasi valutazione recepita nella dichiarazione dei redditi, in quanto ciò fu conseguenza di una scelta legislativa ben esplicitata nel disegno di legge e con la quale si vollero evitare conseguenze penali da valutazioni inadeguate $\mathrm{o}$ comunque in qualche modo discutibili alla luce della complessa normativa tributaria. Come è noto, però, tale scelta è stata poi espressamente superata dal legislatore in occasione della riforma dei reati tributari del 2000, con la quale non solo la menzionata formula è stata modificata, ma specificamente è stata attribuita espressa rilevanza penale alle valutazioni estimative divergenti da quelle ritenute corrette, seppure entro la soglia di tolleranza del 10\% (D.Lgs. n. 74 del 2000, art. 7, comma 2). Come si è detto, la stessa locuzione 'fatti materiali non rispondenti al vero' era stata utilizzata dal legislatore della riforma del 2002, il quale, pure ricorrendo in maniera ( $\mathrm{da}$ alcuni ritenuta) equivoca $[\ldots]$ alla congiunzione 'ancorché', aveva espressamente precisato che oggetto dei 'fatti materiali' potessero essere anche le valutazioni, sostanzialmente recependo fa consolidata interpretazione giurisprudenziale e dottrinaria del termine 'fatti' contenuto nell'originaria formulazione dell'art. 2621".

c) Quindi, per la Corte, il dato testuale e il confronto con la previgente formulazione degli artt. 2621 e 2622, in disarmonia con il diritto penale tributario e con l'art. 2638 cod. civ., sarebbero indubitatamente elementi indicativi della reale volontà legislativa di far venir meno la punibilità dei falsi valutativi, pur rilevando, richiamando opinioni dottrinali resi a riguardo, come non possa del tutto escludersi che l'eliminazione di qualsiasi espresso riferimento a questi ultimi sia da imputarsi alla ritenuta superfluità di una loro evocazione. Tale tesi si fonderebbero soprattutto sul dichiarato timore di una riduzione della portata operativa della normativa e finiscono per fare ricorso soprattutto ad una 
interpretazione sistematica, partendo dall'assunto che le voci di bilancio sono costituite quasi interamente $\mathrm{da}$ valutazioni. Tuttavia - argomenta la Corte - "in tale ottica si dovrebbe ritenere che la riforma abbia inteso affermare che la distorsione nella valutazione di un fatto rilevi nella misura in cui la comunicazione sociale finisca per rappresentare una realtà materiale oggettivamente falsa. E ciò in quanto la valutazione deve considerarsi comunque la misurazione di qualcosa che esiste nella realtà 'materiale', per rimanere in sintonia con l'aggettivo 'materiali' attribuito ai 'fatti'. Sotto tale profilo non si trascura come in effetti la locuzione 'fatti materiali' possa ritenersi solo un'endiadi, giacché mal si comprende quali possano essere i fatti 'immateriali' penalmente irrilevanti. Come si dirà più avanti, però, la scelta della riforma del 2002 di aggiungere l'aggettivo 'materiali' nell'espressione 'fatti non rispondenti al vero' di cui all'art. 2621 cod. civ. previgente, con l'ulteriore richiamo espresso alle 'valutazioni', era stata letta da molti come conseguenza della necessità di risolvere definitivamente $\mathrm{i}$ dubbi interpretativi insorti in relazione alla vecchia formulazione della norma";

d) Ad avviso dei giudici di legittimità occorre fare riferimento ai canoni ermeneutici previsti ex art. 12 disp. gen., comma 1, Cod. Civ.: "Nell'applicare la legge non si può ad essa attribuire altro senso che quello fatto palese dal significato proprio delle parole secondo la connessione di esse, e dall'intenzione del legislatore"; in tale contesto gli stessi affermano essere "arduo pensare che, pur avendo la novella mutuato solo la locuzione 'fatti materiali non rispondenti al vero' della riforma del 2002, epurandola del riferimento specifico alle 'valutazioni', $\underline{\text { si sia ignorato }}$ (oltre che l'esistenza nell'ordinamento delle citate norme che - contenevano e contengono lo stesso riferimento ai 'fatti materiali') il dibattito nel quale gli interpreti a lungo erano stati impegnati nel confronto tra la disciplina introdotta nel 2002 e quella previgente, nonché come si è già detto - l'opinione di chi, subito dopo la suddetta riforma, affermò che l'espressione 'fatti materiali non rispondenti al vero, ancorché oggetto di valutazioni' finiva per sancire quanto dottrina e giurisprudenza prevalenti avevano ritenuto nell'interpretare l'allora previgente dettato normativo, che appunto limitava il riferimento ai 'fatti' non rispondenti al vero. Infatti, è noto come la dottrina e la giurisprudenza avevano dato vita sul vecchio testo dell'art. 2621 cod. civ. ad un articolato dibattito in merito alla rilevanza penale delle valutazioni. Anche la giurisprudenza civile di questa Corte aveva avuto necessità di precisare che l'ipotesi dell'erronea valutazione delle attività nel bilancio sociale se, normalmente, non rientra nel campo della falsità dei bilanci prevista dall'art. 2621 cod. civ., dato che la valutazione importa, necessariamente, un 
apprezzamento discrezionale, in tal campo rientra, invece, allorquando essa oltrepassi il limite di ogni ragionevolezza, non potendo in tal caso parlarsi di discrezionalità, ma di valutazione artificiosa mirante a nascondere gli utili realizzati o a dimostrare l'esistenza di utili che, in realtà, non esistono". (V. 1450-66, massima n. 322888). (Sez. 1, Sentenza n. 484 del 13/2/1969, Rv. 338595). Ed ancora, si era affermato che perché un bilancio di società possa ritenersi falso è necessaria, secondo la dizione dell'art. 2621 cod. civ., la esposizione fraudolenta di fatti non rispondenti al vero o la omissione di fatti concernenti le condizioni economiche della società. Ciò esclude dal campo della falsità l'ipotesi della erronea valutazione delle attività e delle passività sociali, $\underline{\text { dato che }}$ $\underline{\text { tali }}$ valutazioni importano necessariamente un apprezzamento discrezionale, ma tale valutazione non deve oltrepassare il limite di ogni ragionevolezza, poiché in tal caso non potrebbe parlarsi più di discrezionalità $e$ $\underline{\text { si tratterebbe invece di valutazioni }}$ artificiose, che mirano a nascondere gli utili realizzati od a dimostrare come $\underline{\text { esistenti utili che in realtà non sussistano }}$ (Sez. 1, Sentenza 31/5/1966, n. 1450, Rv. 322888). Nella giurisprudenza penale, poi, si era più volte chiarito che, ai fini della sussistenza del reato di cui all'art. 2621 cod. civ., il mezzo per raggiungere la falsificazione (che la legge indica con il termine fatto non rispondente al vero) si identifica in una qualsiasi attività atta ad alterare la situazione obiettiva. Nel concetto di falsificazione non vanno, di regola, comprese le ipotesi di erronea valutazione delle attività e delle passività dell'ente, in quanto tali valutazioni comportano necessariamente un apprezzamento discrezionale; ma tale valutazione non deve oltrepassare il limite di ogni ragionevolezza, poichè in tal caso si tratterebbe di valutazioni artificiose miranti chiaramente a dimostrare come esistenti utili che in realtà non sussistono (Sez. 6, 17/12/1971, n. 1228, Bazan, Rv. 120344; si veda anche Sez. 5, 18/5/2000, n. 8984, Patrucco P. V. ed altri, Rv. 217767, che ha precisato che, in tema di false comunicazioni ed illegale ripartizioni di utili o di acconti sui dividendi, nell'espressione "fatti non rispondenti al vero" contenuta nella norma incriminatrice, vanno ricomprese le stime sul valore di entità economiche non precisamente calcolabili; invero, pur se la prova della non rispondenza al vero appare difficile da raggiungere quando il fatto si configura come operazione dell'intelletto, non avente un concreto parametro di riscontro, non di meno, anche la stima o valutazione deve essere considerata attività fattuale). Ed ancora, si era detto che, agli effetti del delitto di falsità in bilanci, previsto dall'art. 2621 cod. civ., la erronea valutazione delle attività e delle passività della società, comportando detta 
valutazione necessariamente un apprezzamento discrezionale, non assume di regola rilevanza, purchè non superi ogni limite di ragionevolezza, concretandosi in una valutazione artificiosa mirante ad occultare la reale situazione patrimoniale della società (Sez. 5, 14/5/1976, n. 12229, Hussmann, Rv. 134843). Una parte della dottrina aveva finito per sostenere un'interpretazione molto restrittiva del termine "fatti" contenuto nell'art. 2621 c.c., n. 1, escludendo del tutto dagli stessi le valutazioni. Era prevalsa, però, la tesi che riteneva che pure queste ultime potessero rientrare nel generico concetto di "fatti", sostenendone quindi la rilevanza penale.

e) Dunque - afferma la S.C. - come: "In tale tormentato contesto interpretativo, la riforma del 2002 apportò delle parziali modifiche alla condotta, con attribuzione di rilevanza penale all'esposizione di 'fatti materiali non rispondenti al vero ancorché oggetto di valutazioni'. Il riferimento specifico ai fatti 'materiali'e la necessità di precisare, con il ricorso alla congiunzione 'ancorché' (come si è detto, con valore chiaramente 'concessivo'), che in essi vanno ricompresi anche le valutazioni, sono stati evidentemente supportati proprio dalla necessità di superare i contrasti interpretativi originati dalla precedente formulazione della norma e di dovere fissare nell'art. 2621 cod. civ., comma 4 (e nell'art. 2622 cod. civ., speculare comma 8) una soglia di punibilità avente ad oggetto proprio le 'valutazioni estimative', ribadendo così come anche le valutazioni potessero assumere rilievo penale laddove dovessero travalicare i confini della soglia in questione. In tal senso si era espressa, come già ricordato, una parte degli interpreti, secondo la quale la nuova locuzione definitivamente sanciva quanto dottrina e giurisprudenza prevalenti avevano ritenuto nell'esegesi del previgente dettato normativo. Anche le Sezioni Unite di questa Corte, che si sono occupate dei contrasti giurisprudenziali creatisi in sede di prima applicazione della riforma del 2002, nell'affrontare specificamente il problema della continuità normativa, con tutte le implicazioni poste dall'art. 2 cod. pen., ebbero a sottolineare, nel confronto tra vecchia e nuova normativa, che l'esposizione deve riguardare fatti materiali non rispondenti al vero, ancorché oggetto di valutazioni', e non più semplicemente 'fatti non rispondenti al vero" (in motivazione S.U., 26/3/2003, n. 25887, Giordano ed altri, Rv. 224605, che ha chiarito come la nuova formulazione delle norme che prevedono i delitti di false comunicazioni sociali e di bancarotta fraudolenta impropria "da reato societario" ad opera, rispettivamente, del D.Lgs. 11 aprile 2002, n. 61, artt. 1 e 4 non ha comportato l'abolizione totale del reati precedentemente contemplati, ma ha determinato una successione di leggi con effetto parzialmente 
abrogativo in relazione a quei fatti, commessi prima dell'entrata in vigore del citato decreto legislativo, che non siano riconducibili alle nuove fattispecie criminose).

f) Queste le conclusioni cui approda la S.C.: "Fatte queste precisazioni e nella prospettata necessità di verificare in che termini vi sia continuità normativa, il passaggio della recente riforma ad una tipizzazione delle condotta (sia attiva che omissiva) che mutua solo la locuzione 'fatti materiali', legittima l'interpretazione che esclude la rilevanza penale ai fatti derivanti da procedimento valutativo. Se, infatti, si è visto quanto aspro fosse il dibattito allorquando la norma attribuiva rilevanza ai soli 'fatti', è chiaro che ora l'impiego dell'aggettivo 'materiali' finisce per inequivocabilmente escludere ogni sorta di valutazione dalla sfera applicativa della fattispecie. E ciò tanto più se si consideri - come si è già sopra sottolineato - che, in un primo momento, il disegno di legge oggetto dei lavori parlamentari attribuiva rilevanza alle 'informazioni' false, adottando così un'espressione indubbiamente idonea a ricomprendere le valutazioni e sicuramente più corretta avuto riguardo proprio alla normativa in materia di comunicazioni sociali (artt. 2423 e ss. cod. civ.). Insomma, non si può ignorare, in una interpretazione che faccia buona applicazione dei criteri ermeneutici propri della materia penale, il non giustificato revirement nella formulazione della fattispecie, con ritorno alla locuzione 'fatti materiali' (in luogo del riferimento al più ampio ed esaustivo concetto di 'informazioni'), espressamente epurati di quell'aggancio alle 'valutazioni', che invece aveva voluto la riforma del 2002, anche ricorrendo all'esplicita previsione di una soglia di punibilità calibrata proprio su di esse (come si è detto, nei citati art. 2621 c.c., comma 4 e art. 2622 c.c., comma 8). A tal proposito va sottolineata e ribadita l'esigenza di tipicità della norma nella sua applicazione in sede penale, che - come si è visto - nei testi previgenti degli artt. 2621 e 2622 finiva per essere soddisfatta proprio mediante l'individuazione di una soglia di rilevanza delle valutazioni estimative e l'implicito rinvio relativamente alla condotta alla disciplina dettata dall'art. 2426 cod. civ., che appunto fissa i criteri da osservarsi nelle salutazioni. Come è noto, l'interpretazione giurisprudenziale aveva rilevato come le soglie fissate dalla normativa del 2002 non dovessero considerarsi mere condizioni obiettive di punibilità, e quindi 'esterne' alla struttura del reato, ma concorressero proprio a tipizzare le condotte penalmente rilevanti (si veda Corte Cost. n. 161/2004, nonché la già citata Sez. Un., 26/3/2003, Giordano; e in motivazione Sez. 5, n. 44007 del 28/9/2005, Vintaloro ed altro, Rv. 232804). Nella nuova normativa di cui agli artt. 2621 e 2622 la suddetta esigenza di tipizzazione 
della condotta non risulta affatto soddisfatta e il mancato esplicito riferimento alle valutazioni estimative finisce, con una interpretazione estensiva della nozione di 'fatti materiali', per lasciare all'interprete la discrezionalità (e quindi l'arbitrio) di precisarne la rilevanza, in evidente violazione del principio di tipicità del precetto penale. Ciò risulta ancor più pregnante ove si consideri pure l'ulteriore incertezza creata dalla struttura della fattispecie di cui all'art. 2621 cod. civ., nella quale - come si è già evidenziato - i 'fatti materiali' devono anche essere 'rilevanti'; precisazione che invece, inspiegabilmente, non viene replicata nella gemella disposizione di cui all'art. 2622 cod. civ. L'aggettivo 'rilevanti' riferito ai 'fatti materiali' risulta pregno di genericità e in tal modo la determinazione della soglia di penale rilevanza viene ancora una volta lasciata alla valutazione discrezionale del giudice. Peraltro, sempre sotto lo stesso profilo e per quello che in particolare interessa in questa sede, nella quale sono contestati reati di bancarotta impropria da falso in bilancio, l'esigenza di una tipizzazione si fa ancora più intensa ove si consideri che, mentre l'art. 223, comma 2, n. 2 legge fallimentare prevede un reato di evento (costituito dal fallimento) con condotta a forma libera (tanto che si è dubitato della legittimità costituzionale della stessa norma), la fattispecie prevista dall'art. 223, comma 2, n. 1 è un reato anch'esso di evento (costituito dal dissesto) ma a condotta vincolata, giacché quest'ultima è definita dal richiamo operato con l'espressione 'commettendo i fatti' previsti dagli artt. 2621 e 2622 (nonché da tutte le altre norme del codice civile espressamente indicate nella stessa norma). Assume, quindi, particolare rilevanza, ai fini del rispetto del principio di tipicità della fattispecie penale, una chiara delimitazione della condotta, per evitare di incorrere in interpretazioni vietate ex art. 14 disp. gen.”. Segue in sentenza paragrafo: “L'applicazione ex art. 2 cod. pen. della nuova disciplina ai casi in esame. L'opzione interpretativa sopra rappresentata, e alla quale si aderisce, richiede la verifica di quali siano, alta luce dei criteri previsti in materia di successione di leggi penali, gli ambiti applicativi della nuova fattispecie di false comunicazioni sociali (i cui 'fatti', si ripete per quanto qui di interesse, sono richiamati dall'art. 223, comma 2, n. 1 della legge fallimentare), ove si consideri che la maggior parte delle poste di bilancio altro non è se non l'esito di procedimenti valutativi e, quindi, non può essere in alcun modo ricondotta nell'alveo dei soli fatti materiali, come previsti dalla normativa introdotta dalla L. n. 69 del 2015. Certamente la valutazione di qualcosa di inesistente ovvero l'attribuzione di un valore ad una realtà insussistente non può che ritenersi esposizione di un fatto materiale non rispondente al vero. E' ben vero, 
tuttavia, che sono comunque ipotizzabili casi nei quali possa trovare applicazione anche una condotta incentrata sul mendacio ricadente solo su fatti materiali: si pensi ai ricavi 'gonfiati', ai costi effettivamente sostenuti ma sottaciuti oppure alle falsità aventi ad oggetto l'esistenza di conti bancari o a rapporti contemplati da fatture emesse per operazioni inesistenti. Parimenti devono essere ritenute rilevanti pure condotte scaturenti da fatti storici: un esempio è quello dei crediti lasciati in bilancio sebbene ormai definitivamente inesigibili per il fallimento senza attivo del debitore; altro ovvio esempio è quello della omessa indicazione della vendita o dell'acquisto di beni. E 'fatto materiale' può considerarsi anche la mancata svalutazione di una partecipazione nonostante l'intervenuto fallimento della società controllata $\mathrm{O}$, ancora, l'omessa indicazione di un debito derivante da un contenzioso nel quale si è rimasti definitivamente soccombenti".

La Cassazione, dunque, osservato quanto sopra e tenuto conto della nuova formulazione dell'art. 2621 cod. pen. (i cui fatti sono richiamati dall'art. 223, comma 2 , n. 1, 1. fall.), ritenuta applicabile al caso che ha esaminato ex art. 2 cod. pen. nella parte in cui risulta più favorevole, ha annullato in parte qua la sentenza senza rinvio perchè $i$ fatti non sono più previsti dalla legge come reato.

Deve essere detto però che, di recente, altra sentenza della medesima Sezione, della quale è disponibile al momento solo l'informazione provvisoria $^{11}$, ha espresso, sul punto, orientamento contrario.

Con riferimento alla questione esaminata, "Se, a seguito, della modifica dell'art. 2621 cod. civ., introdotta dall'art. 9 legge 27.5.2015 n. 69 anche mediante la soppressione dell'inciso 'ancorché oggetto di valutazioni', il falso c.d. valutativo sia tuttora punibile", la Cassazione ha adottato la seguente Soluzione Affermativa: Nell'art. 2621 c.c. il riferimento ai 'fatti materiali' quali possibili oggetti di una falsa rappresentazione della realtà non vale a escludere la rilevanza penale degli enunciati valutativi, che sono anch'essi predicabili di falsità quando violino criteri di valutazione predeterminati o esibiti in una comunicazione sociale. Infatti, quando intervengano in contesti che implicano l'accettazione di parametri di valutazione normativamente determinati o, comunque, tecnicamente indiscussi, gli enunciati valutativi sono idonei ad assolvere una funzione informativa e possono dirsi veri o falsi".

Tale decisione, resa all'udienza del 12/11/2015, della quale allo stato - come detto - non si conoscono le motivazioni - segue alla citata Relazione del Massimario (Rel.: V/003/15).

Si ritiene, in attesa di esaminare la motivazione, che tale Relazione possa aver determinato il convincimento espresso dai giudici di legittimità, contrario a quello reso nella sentenza "Crespi".

Nella Relazione il Redattore Silvestri giunge infatti alle seguenti Considerazioni riepilogative: "Le considerazioni conseguenti alla lunga ricostruzione effettuata sono nel senso che:

\footnotetext{
11 Informazione Provvisoria n. 13/2015 - Udienza P.U. 12.11.2015 - Presidente: Aniello Nappi - Relatore: Paolo Antonio Bruno - Ricorrente: Flavio Giovgnoli - P.M.: dott. Gabriele Mazzotta - Questione esaminata: Se, a seguito, della modifica dell'art. 2621 cod. civ., introdotta dall'art. 9 legge 27.5.2015 n. 69 anche mediante la soppressione dell'inciso "ancorché oggetto di valutazioni", il falso c.d. valutativo sia tuttora punibile. Riferimenti normativi: art. 2621 cod. civ., art. 223 comma 2, n. 1 legge fall. - Precedenti giurisprudenziali: sent. V Sezione Penale n. 33774/15 Crespi+10.
} 
a) dovendo escludersi la possibilità di accordare alla non riproposizione del sintagma 'ancorché oggetto di valutazioni' una qualsiasi valenza idonea ad eliminare le valutazioni dall'ambito di applicabilità delle nuove disposizioni in materia di false comunicazioni sociali;

b) dovendo escludersi la possibilità di attribuire alla locuzione 'fatti materiali' un significato più restrittivo sia rispetto a quello di 'fatti';

c) dovendo escludersi la possibilità di attribuire alla locuzione 'fatti materiali' un significato selettivo rispetto a quello di 'informazioni;

il riferimento ermeneutico più affidabile rimane quello elaborato sotto la vigenza dell'originario dettato dell'art. 2621 cod. civ. e cioè, che : 'si ha falsità penalmente rilevante solo nei casi in cui le informazioni (offerte dal bilancio) sono il frutto di una valutazione che falsifica o l'entità quantitativa del dato di riferimento (...) oppure (o anche, poiché sono possibilità non alternative) lo valuta impiegando un criterio difforme da quello dichiarato e oggi trova normalmente riscontro nella nota integrativa, in contrappunto alle disposizioni di legge». In tal senso l'aggettivo 'materiali' non apporterebbe alla fattispecie un contributo semantico tale da mutare intrinsecamente il senso contenutistico del sintagma e avrebbe solo l'effetto di escludere le sole opinioni di natura soggettiva, le previsioni, i pronostici (quelle operazioni che nella lettura aziendalistica vengono denominate le stime di bilancio congetturali)".

In conclusione, si è voluto con questo intervento dare conto dei difformi orientamenti resi dalla giurisprudenza di legittimità - sulla questione specifica se il falso c.d. valutativo sia tuttora punibile o meno - che sono emersi all'indomani dell'introduzione della legge 27 maggio 2015, n.
69 (in Gazz. Uff., 30 maggio 2015, n. 124). Disposizioni in materia di delitti contro la pubblica amministrazione, di associazioni di tipo mafioso e di falso in bilancio - che ha riformato il reato di falso in bilancio.

Sia permessa un'annotazione personale. La questione, ad avviso di chi scrive, era prevedibile e prevista dallo stesso legislatore, alla luce dei primi commenti ai disegni di legge presentati in attesa di approvazione parlamentare oggetto di rilievi e critiche sulla questione specifica qui esaminata e, soprattutto, alla luce del dibattito parlamentare dal quale nessun elemento chiarificatore rinviene a far emergere l'intenzione dei responsabili del procedimento legislativo, espresso dalla maggioranza dei votanti, i quali anzi, sordi alla richiesta di emendamenti, dichiaratamente demandano la soluzione del problema alla Corte di cassazione ${ }^{12}$.

\footnotetext{
${ }^{12}$ Legislatura $17^{\mathrm{a}}$ - Aula - Resoconto stenografico della seduta n. 420 del 31/3/2015 (Senato) discussione dei disegni di legge; "Intervento Senatrice Mussini (MistoMovX): ..Un altro aspetto che trovo estremamente rilevante è il fatto che sia stata inserita l'espressione 'fatti materiali' al posto di 'informazioni', perché l'espressione 'fatti materiali rilevanti non rispondenti al vero' esclude tutta la parte relativa alle valutazioni, che tra l'altro spettano proprio ai vertici, nel momento in cui si forma il bilancio. Escludere le valutazioni e considerare solo i fatti materiali non è sufficiente. Faccio un esempio. Poniamo il caso che io abbia 100 veicoli in un magazzino, ai quali assegno, per mia valutazione, un valore di 10.000 euro l'uno; avrò un valore totale di 1 milione di euro. È chiaro che il fatto materiale consisterebbe nel modificare il numero dei veicoli che ho in magazzino; però, se io vado a modificare il valore dei veicoli stessi, comunico comunque un'informazione falsa, ma non un fatto materiale non rispondente al vero, perché si rimane nell'ambito delle valutazioni. Un altro aspetto è costituito dalla soppressione dell'avverbio 'consapevolmente', così come dalla soppressione dell'espressione 'concretamente idoneo ad indurre altri in errore'. Questa di fatto è un'ulteriore limitazione della possibilità di applicare questa norma, perché occorre che ci sia una condotta decettiva e, allo stesso tempo, questa condotta deve esserlo in concreto. Tra l'altro, non si risolve il problema dell'esatta definizione di colui che viene tratto in errore. Si dice infatti: 'indurre altri in errore'. Altri chi? Tra l'altro, la definizione di questi 'altri' dipende anche dalla capacità di leggere un bilancio e di cadere in errore; dipende quindi dalle qualità soggettive di questi 'altri',
} 
che concretamente dovrebbero essere indotti in errore. $\mathrm{La}$ soppressione dell'avverbio 'consapevolmente' viene chiesta per le stesse ragioni che mi inducono a chiedere la soppressione dell'espressione 'concretamente idoneo ad indurre altri in errore'. Intervento Caliendo (FI-PdL XVII): Signora Presidente, vorrei ricordare a tutti che da sempre nel falso in bilancio, quando si parla di comunicazioni non rispondenti al vero, è sempre stata aggiunta la frase 'ancorché oggetto di valutazioni'. Nel corso dell'esame in Commissione ho chiesto al relatore $\mathrm{e}$ al Governo se, essendo oggetto di valutazione, non è più punibile. Mi è stato risposto che sarà la giurisprudenza a dire sì o no. Vorrei ricordarvi un articolo sul Corriere della Sera, a firma di Ferrarella, il quale dice che dal testo sono state espunte le valutazioni. Per cui, a una lettura letterale, il testo porta ad escludere che le valutazioni possano rientrare nel reato. Rispetto a questa incongruenza e indecisione o genericità della norma, come volete chiamarla, vi chiedo di valutare quale può essere la posizione di un'azienda straniera che deve venire a investire in Italia. Prima di investire, ovviamente vuole rendersi conto di quale sono le norme penali che sovrintendono alla corretta gestione delle informazioni sociali. Nell'ipotesi di specie, la domanda è la seguente: se voi vi trovaste al posto dell'amministratore delegato di quella società, non sareste in grado di dire se la dizione 'ancorché oggetto di valutazioni' sia o meno presente nel testo. Credo che tale dizione non possa non essere inserita. Infatti, quando dipendono da stime, le notizie non risultate veritiere... [....]. [..... Immaginiamo che un determinato fatto, che io devo comunicare dipenda da stime e che, per ipotesi, i miei esperti e consulenti a cui mi sono rivolto siano quattro commercialisti che hanno, nella valutazione, dato una stima di un certo tipo; un domani un pubblico ministero potrebbe contestarla, dicendo: siccome le stime dicevano 100 e il mio consulente tecnico dice 105 , hai commesso il reato. Pertanto, in primo luogo, occorre specificare all'interno del testo se le espressioni non rispondenti al vero comprendono o meno le valutazioni. Poi vedremo, nell'articolo 2621-bis, di cosa si tratta. Nell'ipotesi che le valutazioni siano comprese, dobbiamo porci il problema del limite di scostamento, altrimenti tutto è punibile. Vogliamo affossare definitivamente l'economia italiana già in crisi? Vogliamo mettere le piccole imprese nell'impossibilità di funzionare? Liberi di farlo. Per il momento, signora Presidente, mi limito a dire che comunque questa precisazione è necessaria all'interno del testo. [...]. Risposta di D'Ascola, relatore: Ora, il paradosso è costituito, tra l'altro, dalla circostanza che molti illustri senatori si lamentano che il fatto è iperpregnante. Abbiamo obiezioni che si lamentano che sia stata usata l'espressione 'fatti materiali'; si lamentano del 'concretamente idoneo'; si lamentano del 'consapevolmente', ossia di tutte quelle componenti dimostrative di una evidente pregnanza fattuale della componente oggettiva come del suo versante soggettivo. Voglio dire che il fatto è un fatto iperdeterminato e che l'attributo di rilevanza non è un elemento quantitativo indeterminato, ma un elemento ulteriormente confermativo della pregnanza sul piano della offensività della condotta dal momento che rilevante significa qui effettivamente dotato di carica di offensività. Già due volte ho dato una spiegazione - ci mancherebbe altro, ovviamente ognuno è libero di poter dire che queste spiegazioni non convincono; nessuno ha la pretesa di convincere gli altri - ma adesso lo faccio per la terza volta: 'rilevante' significa un fatto materiale dotato di una capacità concretamente offensiva dell'interesse giuridicamente protetto. Questo lo si fa nel solco di una giurisprudenza della Corte costituzionale che riconosce il principio di offensività in due dimensioni tra di loro concorrenti: in senso astratto, perché la norma deve essere popolata di elementi dimostrativi della astratta offensività della condotta; in senso concreto, perché questo richiamo contenuto nel testo della norma impone al giudice una interpretazione che noi qualifichiamo come orientata all'offesa. Quindi, rilevante vuol dire offensivo dell'interesse giuridicamente protetto, ovviamente nel solco di una consolidata giurisprudenza della Corte costituzionale. D'altronde, come ho già detto e mi permetto di invitare i colleghi senatori a riflettere, poi certamente a dissentire - 'rilevante' è attributo non di un elemento quantitativo. Qui nessuno ha scritto variazioni, entità, quantità rilevanti; qui si è scritto 'fatti materiali rilevanti' e il principio di materialità, evocato con assoluta chiarezza dall'attributo 'materiali', è connesso ai fatti, e quindi a dimensioni ontologiche e non a categorie dello spirito. Quando si parla di fatti si indica una realtà ontologica, una circostanza che è dotata di una sua materialità, per l'appunto; si evoca un principio, quello di materialità, che storicamente è connesso nel diritto penale alla offensività. Quindi, noi diciamo, per ben tre volte, che ci deve essere un fatto, quindi un'entità dotata di consistenza ontologica; che questo fatto deve essere materiale, e che per di più deve essere un fatto offensivo. Allora non si può dire che questa è una condotta indeterminata. Qui non stiamo parlando di alcunché che riguarda l'evento perché sono reati di pura condotta, tra l'altro costruiti sul pericolo; l'evento è una circostanza possibile, ma non necessaria al fine della sussistenza degli elementi del tipo legale. Si è parlato contro il dolo specifico, dicendo che è difficile provarlo. Queste sono affermazioni che vanno sfatate una volta per tutte. Dei problemi probatori non si può far carico certamente il legislatore, ci mancherebbe altro: il problema probatorio è un problema che dà serietà $\mathrm{e}$ consistenza, senatrice Mussini, alla norma penale ed incriminatrice, perché le conferisce quel requisito di concretezza che impone agli organi titolari dell'azione penale di dimostrare le peculiarità e le specificità della norma penale incriminatrice. Dire poi che sono elementi difficili da provare significa ignorare che il furto è costruito sul dolo specifico, altrimenti non avremmo mai potuto condannare nessuno per furto perché c'è scritto 'al fine di trarre profitto'. Sono cose di una tale evidenza che il relatore pensava di non doverne parlare. A proposito poi dell'espressione 'al fine di conseguire per sé o per altri un ingiusto profitto', si chiede chi siano gli 'altri'. Gli altri sono persone diverse dall'autore del fatto, ma questo fa parte della grammatica giuridica, non è necessario intervenire addirittura davanti al Senato per spiegare che 'altri' significa persone diverse dall'autore del fatto, tant'è che la legge dice, proponendo una opposizione tra i due termini, o per sé o per persona diversa da sé: questo vuol dire 'altri'. Non penso che dobbiamo specificare che si tratti di Giovane Rossi o di Mario Bianchi. Questa, peraltro, è un'espressione che sta nel codice dal 1930 e non possiamo meravigliarci di espressioni collaudate da 85 anni di applicazione del diritto penale, perché costituisce ovviamente un vero e proprio paradosso. Venendo all'ingiusto profitto, anche 
qui francamente potrei esimermi dal dire che questa espressione sta nel codice penale da sempre, chiaramente per quei reati rispetto ai quali il legislatore, nell'ambito di una discrezionalità che non gli può essere negata (se c'è qualcuno che pensa che le norme penali si scrivano con il computer mi permetto di dire che non è così) non segue delle categoria preontiche che gli impongano da una parte o dall'altra di orientarsi per come taluno pretende che il legislatore si debba orientare. Il legislatore fa delle scelte, peraltro il dolo specifico significa mirare ad un fine, la realizzazione del quale non è richiesta ai fini della sussistenza del fatto di reato, quindi è sufficiente dimostrare che taluno abbia agito ad un fine, ma poi quel fine non è necessario che si sia realizzato perché il reato sia punibile. Tant'è che noi diciamo che il dolo specifico è un arretramento del momento della punibilità, cioè finisce per determinare un allargamento del campo di applicazione della norma penale incriminatrice. Dire poi che in una perdita di bilancio non c'è un profitto è una cosa che francamente non riesco a capire, perché è chiaro che l'occultamento di una perdita determini un vantaggio per il soggetto che si avvale di questa mistificazione, di questo nascondimento: se una perdita non la si porta in bilancio, questo è un vantaggio. È chiaro che $\mathrm{i}$ concetti non sono antitetici, ma anzi perfettamente consonanti l'uno rispetto all'altro. $\mathrm{Ci}$ si lamenta poi di questi diversi livelli. Le norme - mi sia consentito - vanno interpretate e capite e quanto allinterpretazione, approfitto per anticipare un concetto: nessuno può pensare che noi oggi in Senato finiamo per indovinare quale sarà il percorso giurisprudenziale che verrà assegnato all'articolo 2621, all'articolo 2622, all'articolo 2621-bis o all'articolo 2621ter. Noi riteniamo di avere scritto, e di avere prima ancora pensato, delle norme ragionevoli e conformi ai nostri principi. Ovviamente dobbiamo, come in ogni caso del genere, prefigurare che ci saranno dei tribunali, delle corti d'appello una Corte di cassazione le quali poi daranno risposta ai nostri dubbi. A noi basta aver scritto delle norme che si inseriscono in un quadro di natura costituzionale. Bene, noi abbiamo fatto un fatto base, un fatto materiale offensivo. Rispetto a questo fatto, in via scalare e subordinata, come valore successivo in un ordine gerarchico che vede al primo posto inserita la categoria dei fatti materiali rilevanti, abbiamo i fatti di lieve entità. Illustri senatori, i fatti di lieve entità stanno nel sistema penale da sempre. Noi abbiamo addirittura la possibilità di dire che l'elemento indeterminato, allorquando faccia parte di una norma favorevole, non pone alcun problema di legittimità costituzionale, per come è collaudato nel nostro sistema penale. I fatti di lieve entità stanno nell'articolo 73 - giusto per citarne uno - del testo unico in materia di stupefacenti (decreto del Presidente della Repubblica n. 309 del 1990). Sotto questi fatti di lieve entità ci sono i fatti di tenue offensività, con un gradualismo che - mi sia consentito dirlo con un'unica parola - si capisce: c'è il fatto di lieve entità, che è un fatto offensivo, e poi un fatto offensivo tenuamente tale; c'è un ordine scalare che sta anche nel nostro sistema penale. Il senatore Caliendo ha posto ripetutamente questo problema: insomma, le valutazioni stanno o non stanno dentro i fatti materiali rilevanti? Qui non do la mia interpretazione, non perché non abbia una risposta (perché io una risposta ce l'ho), ma non credo che il relatore debba dare una sua interpretazione. Questo è un problema interpretativo e qui non c'è da scandalizzarsi, perché sappiamo bene che i giudici interpretano le norme.
Il relatore è convinto di quello che dice... (Commenti del senatore Caliendo) ...ma è fuor di dubbio che la teoria della interpretazione si inserisca... Presidente: Senatore Caliendo, lasci parlare il relatore. D'Ascola, relatore. La nostra Corte di cassazione dovrà valutare se gli elementi valutativi e le stime possano o meno rientrare allinterno di un concetto che implica fatti materiali rilevanti. Ripeto, il relatore a tal proposito non dà alcuna risposta, perché non la deve dare; ci mancherebbe altro che la desse. Noi non siamo la Corte di cassazione che dà risposte giurisprudenziali; questo è un tema che la giurisprudenza affronterà. Ripeto, il relatore ha la sua risposta, ma non avrebbe alcuna efficacia e alcun significato vincolante, pertanto il relatore ritiene non di non poterla dare, bensì di non dover dare alcuna risposta su questo aspetto. 'Consapevolmente': io l'ho anche detto: l'avverbio 'consapevolmente' ha una doppia funzione: la funzione di... (Brusio). Signora Presidente, il relatore parla anche per ore, però è un paradosso che taluni si lamentino e pongano questioni e poi... Presidente. Senatore D'Ascola, vedo l'Aula con una tenuta di attenzione... Senatore Romani, la pregherei di aiutare l'Assemblea a procedere nei suoi lavori. Abbiamo ascoltato la sua replica molto puntuale, senatore D'Ascola. D'Ascola, relatore. Mi taccio, perché parlare in questo contesto non ha senso. Presidente. Se lei lo ritiene, sviluppi i suoi argomenti perché credo che riguardino una parte delicatissima del provvedimento. D'Ascola, relatore. Quando l'Assemblea sarà meno stanca, riprenderò le spiegazioni nate dalle obiezioni fatte, ma mi pare che procedere in questo contesto sia perfettamente inutile. Presidente. Inutile non è, perché su un tema molto delicato i lavori preparatori sono sempre rilevanti e quello che lei dice viene registrato. Purtroppo, i senatori sono stati richiamati molte volte. D'Ascola, relatore. Se mi è consentito, signora Presidente, in sede di emendamenti darò per la quarta volta le stesse spiegazioni. Presidente. Dal momento che mi sembra che oggi abbiamo lavorato speditamente e che l'impostazione della questione trattata dagli articoli 8 e 9 sia stata avviata, e mi risulta che ci sia un'intesa sostanziale dei Gruppi per riprendere la discussione del provvedimento domani mattina, credo che, sulla base della sua ampia replica, forse i pareri sarebbe opportuno esprimerli domani. Rinvio pertanto il seguito della discussione dei disegni di legge in titolo ad altra seduta".

Riprende la Seduta Legislatura $17^{\mathrm{a}}$ - Aula - Resoconto stenografico della seduta n. 421 del 1/4/2015: "Caliendo (FI-PdL XVII): Signor Presidente, ieri il relatore con la sua bravura ha tentato di giustificare il fatto che all'assenza dell'espressione 'ancorché oggetto di valutazioni' sopperirebbe il giudice alla cui interpretazione è rimesso stabilire o meno le 'valutazioni'. Nello stesso tempo, ci ha detto che 'fatti materiali rilevanti' sono fatti importanti, quindi il termine rilevante non indicherebbe la misura, e che vi possono essere fatti importanti rapportati alla lieve entità di cui all'articolo 9 rendendo evidente che vi è una fascia di non punibilità; perché, seppure «importanti», quindi non misurati, sta a significare che i fatti hanno un certo rilievo rispetto alla lieve entità. Che cosa avviene per quelli che, invece, sono fatti normali di violazione della norma? All'articolo 9 ho presentato alcuni emendamenti. Non si tratta di soglie qualsiasi. Innanzitutto nell'emendamento 9.317 propongo di inserire, dopo le parole 'il giudice valuta' le seguenti: 'e le falsità o le omissioni non hanno 
determinato un'alterazione sensibile del risultato economico di esercizio'. Nell'emendamento successivo 9.318 propongo di inserire le seguenti parole: 'un'alterazione sensibile del risultato economico di esercizio non superiore al 4 per cento dei ricavi e proventi dell'attività caratteristica'. Ciò vuol dire avere quel minimo di scostamento tra valutazioni che possono essere effettuate da un determinato tecnico (tutti sapete che le valutazioni possono oscillare dagli uni agli altri) e avere qui una determinazione precisa di quella che è l'alterazione del risultato economico di esercizio tale da determinare la certezza. Signor Presidente, lei mi insegna che se un'impresa straniera vuole venire ad investire nel nostro Paese, si informerà sul sistema penale e sulla legislazione che consentono a quella società di operare. Si rivolgerà ad un tecnico, ad un avvocato. L'avvocato cosa risponde? Stando alle parole del relatore, dipenderà dall'interpretazione della giurisprudenza. Quanto al nostro compito, si è detto ieri che non compete a noi dare interpretazione. No! A noi compete dare certezza alla norma penale, alla condotta, all'evento, al dolo e a tutti gli elementi della fattispecie. La certezza è tale da incidere sul problema della vita delle aziende e sul problema della vita degli investimenti che le società vogliono fare in Italia. Quindi, ci troviamo davanti ad una fattispecie che viene integrata ad libitum da qualsiasi giudice. Ognuno farà le proprie valutazioni sulla rilevanza $o$ meno. Aspetteremo anni - lei mi insegna - per avere una giurisprudenza sedimentata o un pronunciamento delle sezioni unite della Cassazione che ci diranno cosa vuol dire 'rilevanti'. Mi si deve dunque spiegare perché non individuare dei livelli chiari e precisi di non punibilità. Le soglie attualmente vigenti sono state ritenute conformi e legittime dalla Corte di giustizia europea e dalla Corte costituzionale italiana. Anzi, la nostra Corte costituzionale ha detto una cosa chiara e sacrosanta: esse servono a delimitare il potere di interpretazione dei giudici e a dare contezza e certezza del precetto $\underline{\text { normativo, }}$ che devono essere mantenute. Non intendo mantenere le soglie attualmente vigenti e, anche per quanto riguarda le valutazioni estimative, non parto da una differenza del 10 per cento rispetto a quella che si ritiene corretta, ma propongo di ridurre tale differenza all' 8 per cento, mentre l'emendamento di cui vi ho appena dato lettura si riferisce ad una variazione dei ricavi non superiore al 4 per cento. Signor Presidente, abbiamo scelto una strada che non è di ostruzionismo e non abbiamo fatto emendamenti volti alla riduzione della pena, che il Governo ha voluto elevare, seguendo anche l'indicazione del disegno di legge a prima firma del presidente Grasso, che quindi abbiamo condiviso. Stiamo invece ragionando sulla certezza del precetto penale e abbiamo ragionato su tutta questa normativa. Ha visto che finalmente qualcuno degli emendamenti è stato approvato: sono stati approvati quattro emendamenti in Commissione e un altro ieri ha raggiunto l'unanimità. Forse sarebbe stato giusto approvare un altro emendamento, ovvero quello che avrebbe concesso maggiori poteri all'Autorità anticorruzione. Signor Presidente, proprio ieri, in un convegno, ha messo in evidenza come la corruzione sia un problema che va combattuto, specialmente attraverso la prevenzione. $\mathrm{Su}$ questo abbiamo offerto collaborazione, ma non troviamo ascolto, perché si vuole approvare una norma manifesto. $\mathrm{Su}$ questo aspetto, che riguarda aspetti tecnici di definizione della fattispecie penale, si propone quindi di tornare a delle soglie, che non sono quelle attualmente vigenti, ma che hanno la possibilità di essere valutate concretamente, determinando finalmente una certezza. Vede, signor Presidente, se dobbiamo approvare una norma che non avrà efficacia immediata, perché dobbiamo attendere l'interpretazione della giurisprudenza al fine di dare la valutazione dell'esatta portata, tutto ciò diventa abbastanza ridicolo. Abbiamo fatto una corsa per approvare una nuova legge anticorruzione e poi non ne determiniamo un'efficacia immediata. Per questa ragione, signor Presidente, credo che il Governo e il relatore debbano concedere un minimo di attenzione - non chiedo una grande attenzione - valutando concretamente il nostro contributo, che serve a rendere la norma efficace e ad avere una reale capacità di deterrenza. Non posso avere deterrenza da una norma la cui interpretazione sarà rimessa alla giurisprudenza. È evidente - come lei mi insegna, signor Presidente, avendo fatto come me il magistrato per tanti anni - che la prima regola per avere la capacità di incidere sui fenomeni è che il legislatore approvi una norma che abbia certezza: così invece non inciderete. Vi prego dunque di riflettere e di dare una spiegazione del perché non volete individuare una soglia: se una spiegazione non c'è, vuol dire che avete fatto una norma manifesto, ovvero che volete soltanto sbandierare una norma senza raggiungere alcun risultato. ...; segue ancora Caliendo (FI-PdL XVII): Signor Presidente, sull'emendamento 8.316 abbiamo avanzato la richiesta di voto segreto, che ribadiamo. Credo rientri nei suoi compiti, Presidente, invitare l'Aula a dare un'interpretazione alla norma. Ieri, infatti, il relatore ha detto che non compete al Senato stabilire se le 'valutazioni' fanno o meno parte dei fatti non rispondenti al vero. Ancorché la norma contenga tale dizione ormai da anni, quindi qualsiasi azienda, anche straniera potrebbe ritenere che, come ha detto il Corriere della Sera, secondo la dizione letterale, una eventuale valutazione sbagliata sembra non rientrare più nel reato. Noi non possiamo rimettere il giudizio alla giurisprudenza perché, se diciamo questo, veniamo meno al nostro compito: il giudice, quando dovrà interpretare la norma, consulterà i lavori parlamentari e non troverà una soluzione al suo dilemma, ma leggerà che è il giudice stesso ad avere la responsabilità di interpretare come vuole la norma. Vi rendete conto delle abnormità commesse nel corso dei lavori di questo Senato? Signor Presidente, io credo che lei abbia il dovere di chiedere ai Gruppi di esprimere la propria opinione. Se i Gruppi continuano a schiacciare soltanto il tasto rosso o verde del meccanismo di votazione senza dare una motivazione, si rendono complici di quella interpretazione che vuole rimettere la decisione al giudice. Credo che lei debba invitare i Gruppi ad assumere una posizione, perché qualsiasi giudice guarderà i lavori parlamentari per l'interpretazione e da essi non può risultare che la decisione non compete a noi, ma al giudice. Per questa ragione, Presidente, credo sia necessario avere un minimo di responsabilità per la dignità di questa Aula e del Parlamento e per lo svolgimento del lavoro che gli compete, cioè dare norme chiare e precise. Non possiamo delegare ad altri un nostro compito, perché rischiamo addirittura che la norma diventi incostituzionale, secondo quanto la stessa Corte costituzionale ci ha sempre insegnato. Non può essere il giudice a delimitare la fattispecie penale. Nell'ipotesi di specie voglio sapere da ciascun Gruppo, che si assume così la propria 
responsabilità politica, se ritiene di rimettere al giudice l'interpretazione: le valutazioni rientrano o non rientrano? [....]; segue Mussini (Misto-MovX): Signor Presidente, chiedo al senatore Caliendo il permesso di aggiungere la mia firma all'emendamento 8.316; segue Barani (GAL (GS, LA-nS, MpA, NPSI, PpI)): Signor Presidente, vorrei lasciare traccia di quanto già correttamente espresso dal senatore Caliendo, che credo anche il senatore Susta ci abbia voluto dire con l'emendamento precedente e che il senatore Divina ci ha spiegato con parole meno tecniche, ma molto efficaci. Il Governo ha deliberatamente cancellato e messo la scolorina - è un Governo che, con le scolorine, ci sa fare - sulla locuzione 'ancorché oggetto di valutazioni'. Vi ricordo e le ricordo, signor Presidente, che questa locuzione oggi è presente negli articoli 2621, 2622 e 2638 del codice civile e che, quindi, il legislatore l'aveva ovviamente ben espressa. La maggior parte delle false comunicazioni sociali - come lei sa, signor Presidente, a differenza del Governo che non ne è a conoscenza o fa finta di non saperlo - sono costruite su valutazioni. Il 90 per cento delle false comunicazioni sociali é costruito unicamente su valutazioni: la valutazione dei crediti di una banca, l'incaglio, la sofferenza, l'esigibilità, la valutazione del valore di un immobile. Quindi, il Governo e il relatore ci vogliono spiegare in che modo intendono verificare la mancanza di questa formulazione, che era ben espressa nella locuzione previgente degli articoli del codice civile che ho testé elencato e che sono - lo ripeto - il 2621, il 2622 e il 2638 ? Hanno una spiegazione logica plausibile la tengono sotto il cassetto?";

Riprende la Seduta - Legislatura $17^{\mathrm{a}}$ - Aula - Resoconto stenografico della seduta n. 422 del 1/4/2015: "Mussini (Misto-MovX) domanda di parlare per dichiarazione di voto: Come dicevo ieri, la parte relativa al falso in bilancio è sicuramente la più forte e quella che contiene le modifiche più rilevanti. Lasciamo stare le modifiche di cui parlavamo prima sull'inasprimento delle pene; il discorso del relatore di ieri e di questa mattina è stato correttissimo anche nella puntualizzazione lessicale, tuttavia non risolve un aspetto importante e richiamato anche in un articolo di oggi del Corriere della Sera. Vi sono anche una serie di esempi significativi, come i 'fatti materiali rilevanti' rispetto invece a 'informazioni', scelta che forse è anche legata - si dice - a una traduzione dall'inglese, che sicuramente sposta il problema da un discorso più ampio (quello che comprende anche le valutazioni) ad aspetti che sono, appunto, i 'fatti materiali', restringendo quindi l'ambito. Al di là delle osservazioni giustissime fatte dal senatore D'Ascola (che forse parlava anche per la sua personale esperienza come avvocato), in questo caso noi avremmo voluto che l'articolo fosse diverso perché riteniamo che al legislatore spetti il compito di dare un indirizzo certo e chiaro. $\mathrm{Ci}$ premeva, quindi, dare una definizione di «informazioni» che comprendesse tutto e rispetto alla quale comunque il relatore ha confermato nella sua risposta tutta la preoccupazione che ci ha spinti a modificare l'articolo. Egli, infatti, ha ammesso che qui stiamo producendo una norma che necessiterà di interpretazione ben più di quanto, secondo noi, oggi si dovrebbe fare soprattutto in questa materia. È chiaro che un avvocato cassazionista possa avere una sorta di riflesso pavloviano di fronte alla scelta tra una norma che sia più chiara e un'altra che, invece, apra al tripudio della discussione in Cassazione (che, però, spesso è quella nella quale si radunano i
Così, infatti, testualmente afferma il Relatore nel corso della seduta n. 420 del 31/03/2015

(Assemblea Senato): "La nostra Corte di cassazione dovrà valutare se gli elementi valutativi e le stime possano o meno rientrare all'interno di un concetto che implica fatti materiali rilevanti". "La nostra Corte di cassazione dovrà valutare se gli elementi valutativi e le stime possano o meno rientrare all'interno di un concetto che implica fatti materiali rilevanti. Ripeto, il relatore a tal proposito non dà alcuna risposta, perché non la deve dare; ci mancherebbe altro che la desse. Noi non siamo la Corte di cassazione che dà risposte giurisprudenziali; questo è un tema che la giurisprudenza affronterà”. Ergo si demanda la risoluzione del problema all'autorità giurisdizionale.

clienti privilegiati). Noi, invece, avremmo voluto che, rispetto a questo aspetto, fosse stata data un'indicazione certa e chiara e che, come legislatori, ci fossimo assunti la responsabilità di non rimandare alle aule dei tribunali, alla capacità di bravi avvocati o alla responsabilità dei giudici quella che secondo noi avrebbe dovuto essere una certezza e una chiarezza, anche per via dell'importanza della fattispecie del falso in bilancio. Chi vuole - e non perdo tempo a richiamarlo - può anche andare a vedere sui giornali on line di oggi pomeriggio quanti sono i casi di clamorose situazioni nelle quali il falso in bilancio è emerso proprio in relazione a valutazioni e stime. Questo aspetto è rilevante ma non ci impedirà, in ogni caso, di esprimere un voto positivo sulla globalità di questo disegno di legge, pur essendoci noi astenuti su tutti gli articoli che riguardano il falso in bilancio; non ci impedirà di esprimere un voto positivo su questo disegno di legge che però, sicuramente, non contiene la globalità dell'approccio che noi, come Senato e come legislatori, avremmo voluto dare, anche in relazione a quello che è stato detto prima, durante la discussione generale, e anche dal senatore Monti in apertura...;

Caliendo (FI-PdL XVII): Sul falso in bilancio avevamo proposto pochi emendamenti, che servivano però a rendere costituzionale quella norma, che invece non solo è incostituzionale perché lascia al giudice l'interpretazione. Non lo dico io, ma lo dice la Corte costituzionale sin dal 1989 . Non possiamo prenderci in giro e parlare di fatti materiali rilevanti, come fossero qualcosa che è al di fuori, dicendo che sono importanti: anche dire ciò significa stabilire un limite tra i fatti materiali rilevanti e quelli di lieve entità....”. 
Invero - esprimo però una opinione personale la delimitazione certa del "vero legale", e dunque la non punibilità del fatto per mere "valutazioni estimative", aveva trovato una corretta regolamentazione nella disciplina previgente laddove si prevedeva, senza necessità di interpretazioni libere da parte dei giudici, che le stesse fossero irrilevanti qualora non differissero in misura non superiore ad una certa percentuale.

In tal modo le soglie di punibilità (del caso il legislatore avrebbe potuto abbassarle) integravano requisiti essenziali di tipicità del fatto ovvero condizioni di punibilità e cioè elemento che delimitava l'area d'intervento della sanzione prevista dalla norma incriminatrice.

Certamente il requisito delle soglie di punibilità non sottraeva determinati fatti all'ambito di applicazione della sanzione penale, valendo le stesse, piuttosto, a precisare l'oggetto del reato.

Peraltro le soglie di punibilità avevano superato il vaglio di costituzionalità13.

13 Cfr. Corte Costituzionale - 161/2004: "Sono inammissibili le q.1.c. dell'art. 11 comma 1 lett. a) n. 11.3 ottobre $2001 \mathrm{n}$. 366, censurato, in riferimento all'art. 76 cost., nella parte in cui - nel dettare i principi e criteri direttivi della riforma della disciplina penale delle società commerciali, oggetto di delega legislativa al Governo prescrive di precisare, quanto alla nuova formulazione del reato di false comunicazioni sociali, 'che le informazioni false od omesse devono essere rilevanti e tali da alterare sensibilmente la rappresentazione della situazione economica, patrimoniale e finanziaria della società o del gruppo al quale essa appartiene, anche attraverso la previsione di soglie quantitative', nonché nella parte in cui prescrive, agli stessi fini, di 'prevedere idonei parametri per i casi di valutazioni estimative', e dell'art. 2621 commi 3 e 4 c.c., come sostituito dall'art. 1 d.lg. 11 aprile 2002 n. 61, che ha dato attuazione ai suindicati criteri di delega, censurato in riferimento agli art. 3, 25 comma 2, 76 e 117 comma 1 cost., e all'art. 8 della convenzione OCSE sulla lotta alla corruzione di pubblici ufficiali stranieri nelle operazioni economiche internazionali, fatta a Parigi il 17 dicembre 1997. Tali questioni, infatti, risultano espressamente finalizzate ad ottenere una pronuncia che - tramite la rimozione delle soglie di punibilità a carattere percentuale - estenda l'ambito di applicazione della norma incriminatrice di cui all'art. 2621 c.c. a fatti che attualmente non vi sono
Conclusivamente: sarebbe auspicabile un intervento delle Sezioni Unite trattandosi di questione di particolare importanza ed, in ogni caso, in considerazione del contrasto, di cui si è dato conto, insorto tra le decisioni della medesima sezione $\mathrm{V}$.

\section{Riferimenti bibliografici.}

- Alessandri A., Diritto penale e attività economiche, il Mulino, Bologna, 2010.

- Bossi C., "False comunicazioni sociali: la norma costruita tra continuità normativa e differenze sostanziali", in Diritto \& Giustizia, fasc. 33, 2015, pp. 34 e ss.

- Bricchetti R., Pistorelli L., "La lenta 'scomparsa' del diritto penale societario italiano", in Guida dir., fasc. 26, 2015, pp. 53 e ss.

- Gambardella M., “Il ‘ritorno’ del delitto di false comunicazioni sociali: tra fatti materiali rilevanti, fatti di lieve entità e fatti di particolare tenuità. The new regulation of the crime of false accounting", Cassazione Penale, fasc. 5, 2015, pp. 1723B e ss.

- Lanzi A., "Quello strano scoop del falso in bilancio che torna reato", in Guida dir., fasc. 26, 2015, pp. 10 e ss.

- Perini A., "I 'fatti materiali non rispondenti al vero': harakiri del futuribile 'falso in bilancio'?", in Diritto Penale Contemporaneo, 27 aprile 2015.

\footnotetext{
compresi, e cioè una pronuncia all'adozione della quale osta l'art. 25 comma 2 cost., il quale esclude che la Corte costituzionale possa introdurre in via additiva nuovi reati o che l'effetto di una sua sentenza possa essere quello di ampliare o aggravare figure di reato già esistenti, trattandosi di interventi riservati in via esclusiva alla discrezionalità del legislatore, senza che possa invocarsi, nella specie, l'orientamento secondo cui sono suscettibili di sindacato di costituzionalità, anche 'in malam partem', le c.d. norme penali di favore, in quanto le soglie di punibilità contemplate dall'art. 2621 c.c. integrano requisiti essenziali di tipicità del fatto ovvero condizioni di punibilità e cioè un elemento che 'delimita' l'area di intervento della sanzione prevista dalla norma incriminatrice, e non già 'sottrae' determinati fatti all'ambito di applicazione di altra norma, più generale, e quindi un elemento che esprime una valutazione legislativa in termini di 'meritevolezza' ovvero di 'bisogno' di pena, idonea a caratterizzare una precisa scelta politico-criminale. Restano assorbiti gli ulteriori profili di inammissibilità delle questioni”.
} 
- Seminara S., "La riforma dei reati di false comunicazioni sociali", in Dir. pen. proc., n. 7, 2015, 7, pp. 813 e ss. 